\title{
Probability distribution of returns in the exponential Ornstein-Uhlenbeck model
}

\author{
G. Bormetti ${ }^{1,2}$, V. Cazzola ${ }^{1}$, G. Montagna ${ }^{3,2,1}$ and O. Nicrosini ${ }^{2,1}$ \\ ${ }^{1}$ Istituto Universitario di Studi Superiori, Centro Studi Rischio e Sicurezza, Viale \\ Lungo Ticino Sforza, 5627100 Pavia, Italy \\ 2 INFN, Sezione di Pavia, Via A.Bassi, 627100 Pavia, Italy \\ ${ }^{3}$ Università degli Studi di Pavia, Dipartimento di Fisica Nucleare e Teorica, Via \\ A.Bassi, 627100 Pavia, Italy \\ E-mail: giacomo.bormetti@pv.infn.it, valentina.cazzola@pv.infn.it, \\ guido.montagna@pv.infn.it, oreste.nicrosini@pv.infn.it
}

\begin{abstract}
We analyze the problem of the analytical characterization of the probability distribution of financial returns in the exponential Ornstein-Uhlenbeck model with stochastic volatility. In this model the prices are driven by a Geometric Brownian motion, whose diffusion coefficient is expressed through an exponential function of an hidden variable $Y$ governed by a mean-reverting process. We derive closed-form expressions for the probability distribution and its characteristic function in two limit cases. In the first one the fluctuations of $Y$ are larger than the volatility normal level, while the second one corresponds to the assumption of a small stationary value for the variance of $Y$.

Theoretical results are tested numerically by intensive use of Monte Carlo simulations. The effectiveness of the analytical predictions is checked via a careful analysis of the parameters involved in the numerical implementation of the EulerMaruyama scheme and is tested on a data set of financial indexes. In particular, we discuss results for the German DAX30 and Dow Jones Euro Stoxx 50, finding a good agreement between the empirical data and the theoretical description.
\end{abstract}

PACS numbers: 02.50.Ey, 05.10.Gg, 05.10.Ln, 05.40.Jc, 89.65.Gh

Keywords: Ornstein-Uhlenbeck processes, Fokker-Planck equations, stochastic differential equations, stochastic volatility models, financial returns, Monte Carlo methods 


\section{Introduction and motivation}

Since the pioneering work of Bachelier [1] about the fair price of derivatives contracts exchanged on the Paris market in the early '900, diffusion processes have been natural candidates for the modeling of the stochastic evolution of financial quantities. Few years later Einstein and, independently, Smoluchowski published their famous works about the diffusion of Brownian particles inside a suspension [2, 3]. Their works were later revisited by Langevin [4], but from a completely different point of view. Indeed he took the point of view of the single particles and derived a description in terms of differential equations governing the microscopic dynamics under the effects of random collisions. What is more important for our work, he also focused on the equation driving the velocity of the diffusing particles and showed the existence of a stationary regime in which the velocity reaches a constant value. He provided the first example of what we currently call in a modern language a mean-reverting process described by a stochastic differential equation (SDE). Similar arguments but in the language of Fokker-Planck partial differential equation can be found in [5, 6]. In financial context it is quite common to meet quantities that by construction can not indefinitely diffuse but reasonably fluctuate around a stationary value. For example, the modeling of interest rate dynamics developed during the '90s entirely deals with mean-reverting rates [8].

Empirical studies have also shown that the volatility, the variable that governs the amplitude of returns fluctuations, is not constant, as postulated by Black and Scholes in their seminal work about option pricing [9]. Indeed, once a suitable volatility proxy is defined, it has been empirically demonstrated that it fluctuates along time switching between regimes with higher and lower activity (bursting effect). Moreover, the assumption of a volatility with a stochastic nature represents a quite effective mechanism responsible for the excess of kurtosis observed in the empirical probability returns distributions. A mean-reverting dynamics is therefore a natural candidate for the modeling of volatility. Indeed in financial literature this idea has been widely exploited, as the introduction of several stochastic volatility (SV) models clearly demonstrates [10, 11, 12, 13, 14, 15]. The research in this field has also been strongly enhanced by the pioneering work of Carr and Madan [16] and, more recently, Lewis [17], through the introduction of Fast Fourier Transform (FFT) numerical techniques. Also the physicists' community has independently analyzed and developed models to capture the stochastic nature of volatility, with particular emphasis on the comparison of the models predictions with real market data. For a review the reader can see [18, while specific analysis are addressed in [19, 20, 21, 22, 23, 24, 25, 26, 27, 28]. Our work follows in particular the guidelines of [20] and [25]. Both these articles present an analytical characterization of the probability distribution of financial returns under the assumption of a Geometric Brownian motion coupled through the diffusion coefficient with a SDE that describes the evolution of the volatility. Dragŭlescu - Yakovenko [20] and Masoliver - Perelló 25] considered two different models, the former authors presenting an analysis for the Heston one [13], while the latter focusing on the Scott 
model [10]. Main results have been derived under the hypothesis that the stochastic process that describes the volatility dynamics has reached a stationary regime, a reasonable assumption if the relaxation time is negligible with respect to the time horizon along with the process evolves. Following Masoliver and Perelló we consider the Scott or exponential Ornstein-Uhlenbeck model, in particular for its well known ability to capture some stylized facts observed in real market data such as squared-returns autocorrelation, leverage effects and multiple time scale properties [22, 25, 26]. However, we extend their analysis relaxing the request of the complete volatility thermalization. A good reason for our choice is that in many financial applications the involved time horizon is comparable with the relaxation time of the process. Moreover, some exotic financial instruments do depend on the entire history of the process. The characterization of the probability distribution, even if in approximated form, in the out-of-stationary regime can provide some interesting insight in the understanding of the process evolution and be of practical interest in the field of quantitative finance.

The structure of the article is the following. In Section 2 we review the SV model we will deal with providing the reader with the formulation in terms of systems of stochastic differential equations. Section 3 and 4 share the same structure. Each of them consists of two subsections, the first one depicts the framework and describes the analytical results, while the second puts the theoretical results on numerical tests. Firstly we present a closed-form expression for the returns probability distribution in the limit case of logvolatility fluctuations higher than the volatility normal level. After providing evidences that the approximate solution is effective only in the limit of small log-volatility variance, we derive an exact closed-formula for the characteristic function but for a linear version of the SV model. Again we check the goodness of the results by means of FFT algorithms and we test them against the Monte Carlo (MC) simulation of the linear and complete models. Section 5 is devoted to the comparison of model predictions with real market data. We discuss in detail the calibration procedure and we apply it on a set of financial indexes from the equity sector. We report more relevant results for the German DAX30 index and the Dow Jones Euro Stoxx 50, a market capitalization-weighted index of 50 European blue-chip stocks from those countries participating in the European Monetary Union. The final Section 6 draws the relevant conclusions and suggests some possible applications of the analytical results in the field of financial option pricing. Some of the results presented in Section 3 are partially shared with a paper [29] appeared on the on-line archive during the completion of this work.

\section{The Model}

The process we are investigating is a slightly modified version of the stochastic volatility model originally introduced by Scott [10]. In financial and econophysics literature it is commonly referred to as an exponential Ornstein-Uhlenbeck process. However, since we allow for a non-null stationary mean value for the random variable driving the logvolatility of the returns, in our case it should be more correct to speak of an exponential 
mean-reverting process. A major difference with Scott model is the existence of a parameter $\rho$, that takes values in $[-1,1]$ and represents the correlation between the two sources of noise in the diffusive process. As we will see later, $\rho$ different from zero is crucial to obtain a non trivial behavior for the skewness of the returns distribution.

Previous considerations translate in the following coupled SDEs

$$
\begin{aligned}
& \mathrm{d} S(t)=\mu S(t) \mathrm{d} t+m \mathrm{e}^{Y(t)} S(t) \mathrm{d} W_{1}(t), \\
& S\left(t_{0}\right)=s_{0}, \\
& \mathrm{~d} Y(t)=\alpha(\gamma-Y(t)) \mathrm{d} t+k \rho \mathrm{d} W_{1}(t)+k \sqrt{1-\rho^{2}} \mathrm{~d} W_{2}(t), \\
& Y\left(t_{0}\right)=y_{0},
\end{aligned}
$$

where $\mathrm{d} W_{1}$ and $\mathrm{d} W_{2}$ are two independent Wiener processes, while $s_{0}, \mu, m, y_{0}, \alpha, \gamma$, $k$ and $\rho$ are constant parameters that can be calibrated on real data. If we define $\sigma(t)=m \mathrm{e}^{Y(t)}$ and we set $m>0$ in Eq. (11) we recognize the same dynamics of a Geometric Brownian motion, with a constant drift coefficient $\mu$ while $\sigma(t)$, the so-called volatility, characterizes the amplitude of the fluctuations of $S$. However, the model does not only allow for a time varying $\sigma$, but through the exponential function promotes the volatility itself to a random variable driven by the dynamics of $Y$. By acting on the value of $k \geq 0$ we can strongly modify the behavior of $Y$. The case $k=0$ switches off the stochastic nature of $Y$ and it evolves deterministically towards its stationary value $\gamma$, with a characteristic time $1 / \alpha(\alpha>0)$. If $k$ is strictly greater than zero, it is well known that $Y$ follows a Gaussian process, with

$$
\begin{aligned}
& \mathbb{E}[Y]=\left(y_{0}-\gamma\right) \mathrm{e}^{-\alpha\left(t-t_{0}\right)}+\gamma \stackrel{t \rightarrow+\infty}{\longrightarrow} \gamma \\
& \mathbb{E}\left[Y^{2}\right]-\mathbb{E}[Y]^{2}=\frac{k^{2}}{2 \alpha}\left[\mathrm{e}^{-2 \alpha\left(t-t_{0}\right)}+1\right] \stackrel{t \rightarrow+\infty}{\longrightarrow} \beta \doteq \frac{k^{2}}{2 \alpha}
\end{aligned}
$$

We have used the notation $\mathbb{E}[\cdot]$ to indicate the expectation with respect to the probability distribution of $Y$, so $\mathbb{E}[Y]$ and $\mathbb{E}\left[Y^{2}\right]-\mathbb{E}[Y]^{2}$ correspond to the usual mean value and variance, respectively. For later convenience, Eq. (1) can be simplified into the following

$$
\mathrm{d} X(t)=-\frac{1}{2} m^{2} \mathrm{e}^{2 Y(t)} \mathrm{d} t+m \mathrm{e}^{Y(t)} \mathrm{d} W_{1}(t),
$$

with boundary condition $X\left(t_{0}\right)=0$, by applying Itô's Lemma to the centered logarithmic return $X(t)=\ln S(t)-\ln S_{0}-\mu\left(t-t_{0}\right)$.

Equations (2) and (5) summarize the model we want to focus on. In particular, once a time horizon $t>t_{0}$ has been fixed, we are interested in the characterization of the returns transition probability distribution $p_{X}\left(X(t) \mid X_{0}, Y_{0}\right)$. The returns distribution $p_{X}(X(t))$ can be readily obtained taking the expectation over the initial distribution of $X_{0}$ and $Y_{0}$. In [25] the authors derive an approximate expression for $p_{X}$ under the assumption of a stationary regime for the $Y$ variable, i.e. normally distributed with mean $\gamma=0$ and variance $\beta>0, p_{Y_{0}} \sim \mathcal{N}(\gamma=0, \beta)$. The further crucial hypothesis assumed by the authors to obtain their results was $\lambda \doteq k / m \gg 1$. This fact was 
supported by empirical evidences based on the analysis of daily Dow Jones Index returns. In the following section we generalize Masoliver and Perelló's result consistently with the boundary condition of Eq. (2), $Y_{0} \sim \delta\left(Y_{0}-y_{0}\right)$, that is relaxing the request of initial stationarity, and allowing for $\gamma$ different from zero. We do not relax the condition for $\lambda$ in order to follow the same solving strategy of the forward Fokker-Planck equation. However we will show the effectiveness of this assumption at the end of the next section providing the results of the numerical MC tests.

Before deriving our results, it can be worth remembering that the distribution of returns is surely not the only interesting statistical feature of mean-reverting SV models and literature deals with other properties, such as squared-returns autocorrelation, leverage effects (i.e. past returns and futures volatilities correlation) and multiple time scale properties [25, 26, 30, 31, 32. However our interest is guided by a possible applications of a closed-form expression for the returns distribution in the field of financial option pricing. Indeed, in a forthcoming work in preparation [33], we will show how the model under consideration here can emerge in a natural way in a risk-neutral description of returns dynamics.

\section{Limit case $\mathbf{I}: k / m \gg 1$}

\subsection{Approximate closed-form expression for the returns distribution}

Given the dynamics (2) and (5), the associated transition probability density function $p\left(x, y \mid x_{0}, y_{0}\right)$ satisfies the forward Fokker-Planck equation [7, 34]

$$
\frac{\partial p}{\partial t}=\frac{m^{2}}{2} \mathrm{e}^{2 y} \frac{\partial p}{\partial x}+\frac{m^{2}}{2} \mathrm{e}^{2 y} \frac{\partial^{2} p}{\partial x^{2}}-\alpha \gamma \frac{\partial p}{\partial y}+\alpha \frac{\partial(y p)}{\partial y}+\frac{1}{2} k^{2} \frac{\partial^{2} p}{\partial y^{2}}+\rho m k \frac{\partial^{2}\left(\mathrm{e}^{y} p\right)}{\partial x \partial y}(6)
$$

with initial condition

$$
p\left(x, y \mid x_{0}=0, y_{0}\right) \doteq p\left(x, y \mid y_{0}\right)=\delta(x) \delta\left(y-y_{0}\right) .
$$

Instead of working with $x, y$ and $t$, it is more convenient to introduce the dimensionless variables

$$
\tau \doteq k^{2}\left(t-t_{0}\right), \quad u \doteq \lambda x \quad \text { and } \quad v \doteq \lambda y .
$$

With respect to $\tau, u$ and $v$ Eq. (6) becomes

$$
\frac{\partial p}{\partial \tau}=\frac{\mathrm{e}^{2 v / \lambda}}{2 \lambda} \frac{\partial p}{\partial u}+\frac{\mathrm{e}^{2 v / \lambda}}{2} \frac{\partial^{2} p}{\partial u^{2}}-\frac{\alpha \gamma \lambda}{k^{2}} \frac{\partial p}{\partial v}+\frac{\alpha}{k^{2}} \frac{\partial(v p)}{\partial v}+\frac{\lambda^{2}}{2} \frac{\partial^{2} p}{\partial v^{2}}+\rho \lambda \frac{\partial^{2}\left(\mathrm{e}^{v / \lambda} p\right)}{\partial u \partial v},
$$

with initial condition

$$
p\left(u, v \mid y_{0}\right)=\delta(u) \delta\left(v-\lambda y_{0}\right) .
$$

The previous relations can be rewritten in terms of the characteristic function

$$
\varphi\left(\omega_{1}, \omega_{2}, \tau \mid y_{0}\right)=\int \mathrm{d} u \mathrm{e}^{i \omega_{1} u} \int \mathrm{d} v \mathrm{e}^{i \omega_{2} v} p\left(u, v \mid y_{0}\right)
$$

The condition $\tau=0$ becomes

$$
\varphi\left(\omega_{1}, \omega_{2}, 0 \mid y_{0}\right)=\int \mathrm{d} u \mathrm{e}^{i \omega_{1} u} \delta(u) \int \mathrm{d} v \mathrm{e}^{i \omega_{2} v} \delta\left(v-\lambda y_{0}\right)=\mathrm{e}^{i \omega_{2} \lambda y_{0}},
$$


while Eq. (8) reduces to

$$
\begin{aligned}
\frac{\partial \varphi}{\partial \tau}\left(\omega_{1}, \omega_{2}, \tau \mid y_{0}\right)= & -\frac{i \omega_{1}}{2 \lambda} \varphi\left(\omega_{1}, \omega_{2}-\frac{2 i}{\lambda}, \tau \mid y_{0}\right)-\frac{1}{2} \omega_{1}^{2} \varphi\left(\omega_{1}, \omega_{2}-\frac{2 i}{\lambda}, \tau \mid y_{0}\right) \\
& +\frac{i \omega_{2} \alpha \gamma \lambda}{k^{2}} \varphi\left(\omega_{1}, \omega_{2}, \tau \mid y_{0}\right)-\frac{\omega_{2} \alpha}{k^{2}} \frac{\partial \varphi}{\partial \omega_{2}}\left(\omega_{1}, \omega_{2}, \tau \mid y_{0}\right) \\
& -\frac{\lambda^{2}}{2} \omega_{2}^{2} \varphi\left(\omega_{1}, \omega_{2}, \tau \mid y_{0}\right)-\rho \lambda \omega_{1} \omega_{2} \varphi\left(\omega_{1}, \omega_{2}-\frac{i}{\lambda}, \tau \mid y_{0}\right) .
\end{aligned}
$$

In order to solve Eq. (12), following [25], we try the ansatz

$$
\varphi\left(\omega_{1}, \omega_{2}, \tau \mid y_{0}\right)=\mathrm{e}^{-A\left(\omega_{1}, y_{0}, \tau\right) \omega_{2}^{2}-B\left(\omega_{1}, y_{0}, \tau\right) \omega_{2}-C\left(\omega_{1}, y_{0}, \tau\right)+\mathrm{O}\left(\omega_{2}^{3}\right)} .
$$

In Appendix $\mathrm{A}$ we show how to obtain the ordinary differential equations (ODEs) satisfied by $A, B$ and $C$ in the limit of small $\omega_{2}$ and up to order $1 / \lambda$. Once the approximate expression of $C\left(\omega_{1}, y_{0}, \tau\right)$ is derived, we can compute the marginal distribution $p_{X}\left(x \mid y_{0}\right)$ noticing that

$$
\begin{aligned}
p_{X}\left(x \mid y_{0}\right) & =\frac{1}{2 \pi} \int_{-\infty}^{+\infty} \mathrm{e}^{-i \omega_{1} x} \varphi\left(\omega_{1} / \lambda, \omega_{2}=0, \tau \mid y_{0}\right) \mathrm{d} \omega_{1} \\
& \simeq \frac{1}{2 \pi} \int_{-\infty}^{+\infty} \mathrm{e}^{-i \omega_{1} x} \mathrm{e}^{-C\left(\omega_{1} / \lambda, y_{0}, \tau\right)} \mathrm{d} \omega_{1} .
\end{aligned}
$$

It is worth observing that $C$ corresponds to the logarithm of the characteristic function, changed by sign. Hence we can easily obtain the cumulant of order $n$ of $p_{X}$

$$
k_{n}=-\left.(-i)^{n} \frac{\partial^{n} C\left(\omega_{1} / \lambda, y_{0}, \tau\right)}{\partial \omega_{1}^{n}}\right|_{\omega_{1}=0} .
$$

This suggests that, instead of computing previous integral directly, we can derive an approximated but closed-form solution by exploiting the Edgeworth expansion [35]. We report below the expansion considering correction to the Gaussian distribution only up to fourth normalized Hermite polynomial [36]

$$
\begin{aligned}
& p_{X}\left(x \mid y_{0}\right) \simeq \frac{1}{\sqrt{2 \pi k_{2}}} \mathrm{e}^{-\frac{(x-k 1)^{2}}{2 k_{2}}} \times \\
& {\left[1+\frac{k_{3}}{\sqrt{6} k_{2}^{3 / 2}} H_{3}\left(\frac{x-k 1}{\sqrt{k 2}}\right)+\frac{k_{4}}{\sqrt{24} k_{2}^{2}} H_{4}\left(\frac{x-k 1}{\sqrt{k 2}}\right)\right] .}
\end{aligned}
$$

The explicit expressions of the first four cumulants are given by

$$
\begin{aligned}
k_{1}= & -\frac{m^{2}}{2 \alpha} \zeta \\
k_{2}= & \frac{m^{2}}{\alpha}\left[(1+2 \gamma) \zeta+2\left(y_{0}-\gamma\right)\left(1-\mathrm{e}^{-\zeta}\right)\right] \\
k_{3}= & 6 \rho \frac{m^{3} k}{\alpha^{2}}\left[\zeta(1+\gamma)+\left(y_{0}-(1+2 \gamma)\right)\left(1-\mathrm{e}^{-\zeta}\right)-\left(y_{0}-\gamma\right) \zeta \mathrm{e}^{-\zeta}\right], \\
k_{4}= & 6 \frac{m^{4} k^{2}}{\alpha^{3}}\left[2 \zeta+\left(1-\mathrm{e}^{-2 \zeta}\right)-4\left(1-\mathrm{e}^{-\zeta}\right)\right. \\
& +4 \rho^{2}\left(\zeta+\zeta \mathrm{e}^{-\zeta}-2\left(1-\mathrm{e}^{-\zeta}\right)\right)
\end{aligned}
$$




$$
\begin{aligned}
& -4 \rho^{2} y_{0}\left(\zeta \mathrm{e}^{-\zeta}-\left(1-\mathrm{e}^{-\zeta}\right)+\frac{1}{2} \zeta^{2} \mathrm{e}^{-\zeta}\right) \\
& \left.+4 \rho^{2} \gamma\left(\zeta+2 \zeta \mathrm{e}^{-\zeta}-3\left(1-\mathrm{e}^{-\zeta}\right)+\frac{1}{2} \zeta^{2} \mathrm{e}^{-\zeta}\right)\right]
\end{aligned}
$$

where we have introduced the ancillary variable $\zeta \doteq \alpha\left(t-t_{0}\right)$. We have checked the consistency of our results when $t$ approaches $t_{0}$ with the initial time condition (7). Indeed, at lowest order in $\zeta, k_{1}$ and $k_{2}$ scale linearly, while being $k_{3}=O\left(\zeta^{2}\right)$ and $k_{4}=O\left(\zeta^{3}\right)$ the skewness $\varsigma \doteq k_{3} / k_{2}^{3 / 2}$ and kurtosis $\kappa \doteq k_{4} / k_{2}^{2}$ do not diverge, as expected.

The analytical expression for $C$ given by Eq. A.10) (and from it, by simple derivation, the explicit characterization of the cumulants) represents one of the main results of this work. However, Eq. (16) has to be considered with care for various reasons. First, it is well known that for an arbitrary choice of $k_{1}, k_{2}, k_{3}$ and $k_{4}$ the positive definiteness of the truncated Edgeworth expansion is not guaranteed. Secondly, when dealing with the problem of probability distribution characterization for the sum of $n$ random variables (e.g. identically distributed and with finite moment of every order) higher order terms of the Edgeworth expansion scales with the power of $n^{-r / 2}$ for suitable $r$. For example the term proportional to $H_{3}$ scales with $n^{-1 / 2}$, while the terms proportional to $H_{4}$ and $H_{6}$ (not considered here) scale with $n^{-1}$. But for the case under consideration we have $n=1$, so in general we can not a priori estimate the goodness of the approximation. Moreover, the entire procedure previously described for the derivation of Eq. (16) is strongly based on some approximations.

It is worth noting that similar approximations have been adopted in [29], where the complete characteristic function is given by Eqs. (35) and (36) yielding, in their notation, the following formula

$$
\begin{aligned}
\varphi(\omega / \lambda, \bar{\alpha} t)=\exp \{ & -i \omega \mu(t)-\left[\bar{m}^{2} t+2 \vartheta\left(t, z_{0}\right)\right] \frac{\omega^{2}}{2}+i \rho \varsigma\left(t, z_{0}\right) \omega^{3} \\
& \left.+\left(\kappa(t)+\vartheta\left(t, z_{0}\right)^{2} / 2\right) \omega^{4}+\mathrm{O}\left(1 / \lambda^{5}\right)\right\},
\end{aligned}
$$

where the various arguments entering the exponential are defined in [29]. Following the procedure of retaining the $\bar{m}^{2}$ term inside the exponential function, while performing a Taylor expansion starting from the $\vartheta$ dependent contribution, the authors of [29] get the approximate expression (Eq. (41) in the same paper)

$$
\begin{aligned}
& \varphi(\omega / \lambda, \bar{\alpha} t)=\exp \left\{-i \omega \mu(t)-\bar{m}^{2} t \frac{\omega^{2}}{2}\right\}\left[1-\vartheta\left(t, z_{0}\right) \omega^{2}+i \rho \varsigma\left(t, z_{0}\right) \omega^{3}\right. \\
& \left.+\left(\kappa(t)+\vartheta\left(t, z_{0}\right)^{2} / 2\right) \omega^{4}+\mathrm{O}\left(1 / \lambda^{5}\right)\right] .
\end{aligned}
$$

This leads to a distribution of returns containing a $H_{2}$ Hermite polynomial contribution as given by Eq. (42) in [29].

On the other hand, if one follows a different approach expanding the exponential starting from the $\omega^{3}$ term, the approximate characteristic function reads, according to the notation of [29], as follows

$$
\varphi(\omega / \lambda, \bar{\alpha} t)=\exp \left\{-i \omega \mu(t)-\left[\bar{m}^{2} t+2 \vartheta\left(t, z_{0}\right)\right] \frac{\omega^{2}}{2}\right\}\left[1+i \rho \varsigma\left(t, z_{0}\right) \omega^{3}\right.
$$




$$
\left.+\left(\kappa(t)+\vartheta\left(t, z_{0}\right)^{2} / 2\right) \omega^{4}+\mathrm{O}\left(1 / \lambda^{5}\right)\right] .
$$

This leads to the probability distribution of the form of Eq. (16), where a $H_{2}$ polynomial term is absent by construction and incidentally this expansion corresponds to Edgeworth series truncated to the fourth Hermite polynomial [35, 36].

In the next section we will discuss numerical tests based on intensive MC simulations in order to check the robustness of our results.

\subsection{Numerical Results}

Standard numerical techniques to simulate random paths from the dynamics Eq. (2) and Eq. (5) are widely discussed in literature and a classical reference is given by [37]. In particular Chapter 6 is dedicated to discrete schemes of SDEs suitable to generate paths using Monte Carlo methods. In our analysis we implement the Euler-Maruyama scheme. The two crucial parameters to be chosen correspond to the discrete time step $\Delta t \doteq\left(t-t_{0}\right) / \mathrm{NSTEP}$ and the total number of MC paths to be generated, MCPATHS. The time variables are measured in yearly units, so $t-t_{0}=1$ corresponds to a diffusion process that evolves for one year. We started with $\Delta t=10^{-1}$ and then decreased it until $\Delta t=10^{-4}$. At the same time we increased MCPATHS from $10^{3}$ to $5 \cdot 10^{7}$. We finally fixed $\Delta t=10^{-3}$ and MCPATHS $=5 \cdot 10^{6}$, because we did not find a significant improvement with smaller value of $\Delta t$.

All the numerical MC results have been obtained with a private Micro Beowulf Cluster made of 4 nodes, each of which is an AMD Athlon 64 Dual Core with $2.00 \mathrm{GHz}$ CPUs and 2.00 GByte of RAM, developed by one of the authors (G.B.) [38]. The resort to 64 bit architecture can be useful to avoid some problem that can arise with high Monte Carlo statistics.

In Fig. 1 we report the returns distributions obtained via $\mathrm{MC}$ simulation and the analytical predictions given by the Edgeworth expansion 16. Curves from bottom to top correspond to increasing values of $\beta$ : $0.5 \%, 1 \%, 2 \%, 5 \%, 10 \%, 25 \%$ and $50 \%$, $\rho=-0.9, m=0.1, \alpha=10, \gamma=0, y_{0}=0$. From the relation $\lambda^{2}=2 \cdot 10^{3} \beta$, we derive $\lambda=3.16,4.47,6.32,10,14.14,22.36,31.62$, that are values consistent with the assumed approximation $\lambda \gg 1$. The choice $\gamma=0$ guarantees to preserve the interpretation of $m$ as the normal level of the volatility in stationary regime. Indeed, $\gamma \neq 0$ introduces an exponential correction which we could easily deal with by a suitable redefinition of $m$ and a linear shift of $Y$. The value $m=0.1$ implies a yearly volatility of the $X$ process of order $10 \%$, while $\alpha$ equal to 10 corresponds to a relaxation time of 0.1 years. In Fig. 1 we consider the time interval $t-t_{0}=0.1$ in order to test the goodness of the analytical approximation in a non-stationary regime. The agreement is quite good for low values of $\beta$ (and therefore low $\lambda$, but always greater than one) and rapidly worsen for higher values for non-stationary regimes. The peak of the $\mathrm{MC}$ distribution moves rightward and the left tail becomes fatter with respect to the analytical prediction as can be clearly seen in the bottom panel of Fig. 1. Indeed for the highest $\beta$ values the Edgeworth expansion starts to oscillate on the tails and becomes negative. In Fig. 2 we 
Figure 1. Returns distributions from MC simulation of the Euler-Maruyama scheme and analytical probability distributions given by Eq. (16). Parameters values as discussed in the text, curves from bottom to top correspond to increasing values of $\beta: 0.5 \%, 1 \%, 2 \%, 5 \%, 10 \%, 25 \%$ and $50 \%$. Curves have been shifted upwards for the sake of readability. Top panel: $\rho=-0.9$ and $t-t_{0}=0.1$; bottom panel: left tails in semi-logarithmic scale.
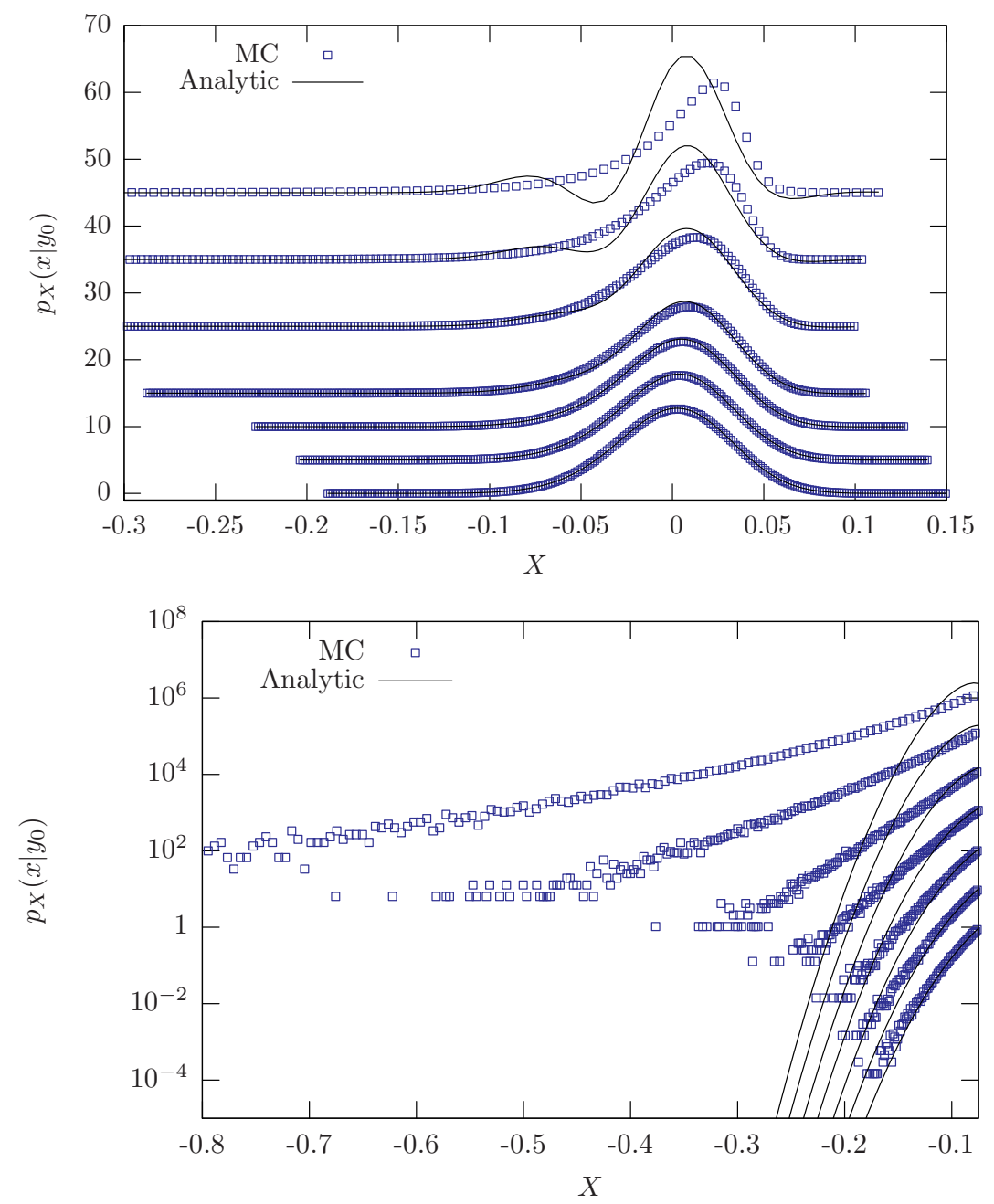

show the behavior of the distribution for a positive value of the correlation coefficient, $\rho=0.5$, and in stationary regime $t-t_{0}=1$. After ten relaxation times the $Y$ process has completely thermalized and again we checked the agreement between $\mathrm{MC}$ based results and the Edgeworth expansion. As correctly predicted by Eq. (19), $\rho$ governs the sign of the skewness and the figures confirm that the asymmetry of the distributions is opposite with respect to the previous case. Again the best agreement corresponds to the lowest $\beta$, while highest values for $\lambda$ are not sufficient to improve it. As in Fig. 1, significant differences are present in the distribution tails.

In order to make our considerations more quantitative, we present in Fig. 3] the scaling of mean, variance, skewness and kurtosis with time. In each panel we report the 
Figure 2. Details as in the caption of Fig. 1. Top panel: $\rho=0.5$ and $t-t_{0}=1$; bottom panel: right tails in semi-logarithmic scale.
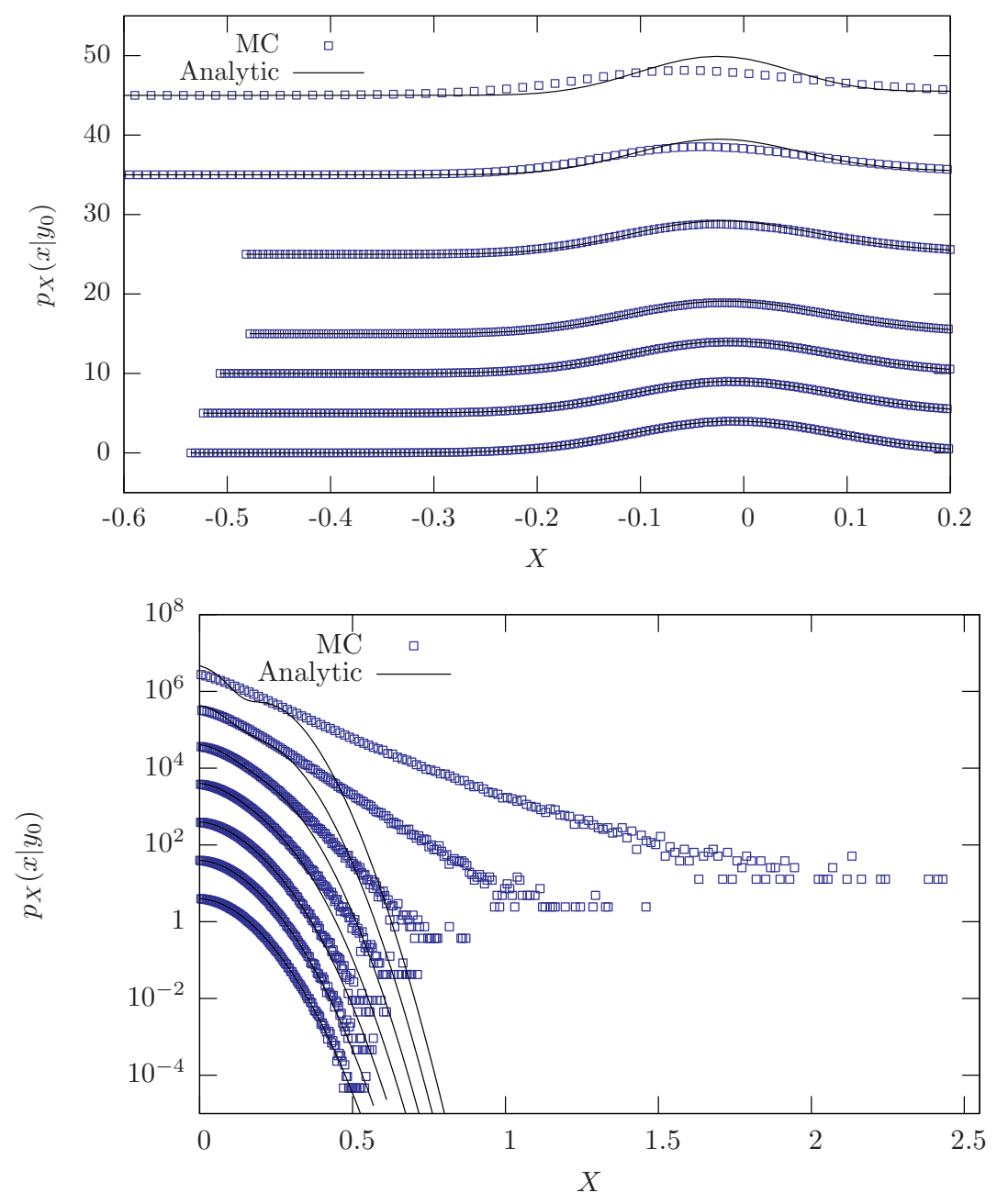

theoretical prediction and the numerical MC results with $95 \%$ error bars for $\beta=0.5 \%$ and $\beta=5 \%$. Tab. 1 contains similar results but for all the $\beta$ considered in Fig. 1 and $t-t_{0}=1$.

For $\beta \leq 10 \%$ mean and variance computed with Eq. (17) and Eq. (18) are in statistical agreement with the MC estimates and the linear scaling with time theoretically predicted is confirmed by the numerical simulations. The compatibility of $\varsigma$ is limited to small values of $\beta$ values while the situation is quite unsatisfactory for $\kappa$ and $\beta \geq 2 \%$. All these empirical evidences strongly suggest that the expansion of Eq. (12) for $\omega_{2}$ nearly zero and up to order $1 / \lambda$ predicts the correct behavior of $C\left(\omega_{1}, y_{0}, \tau\right)$ for small $\beta$ values only. The condition $\lambda \gg 1$ is not enough to guarantee that the Edgeworth expansion (16) is a good approximation of the true distribution. When the stationary variance of the log-volatility becomes high, the fluctuations of $Y$ sensibly deviate from $\gamma$ and the exponential function enhances the fluctuations of $X$. This mechanism is responsible for the growth of the empirical kurtosis at high $\beta$. 
Figure 3. From top left clockwise: scaling with time of mean, variance, kurtosis and skewness. Comparison between numerically estimated values (with MC 95\% confidence level) and theoretical prediction (Eq. (17)-Eq. (20)) for normalized cumulants. $\rho=$ $-0.9, \beta=0.5 \%, 5 \%$ and other parameters as in the text.
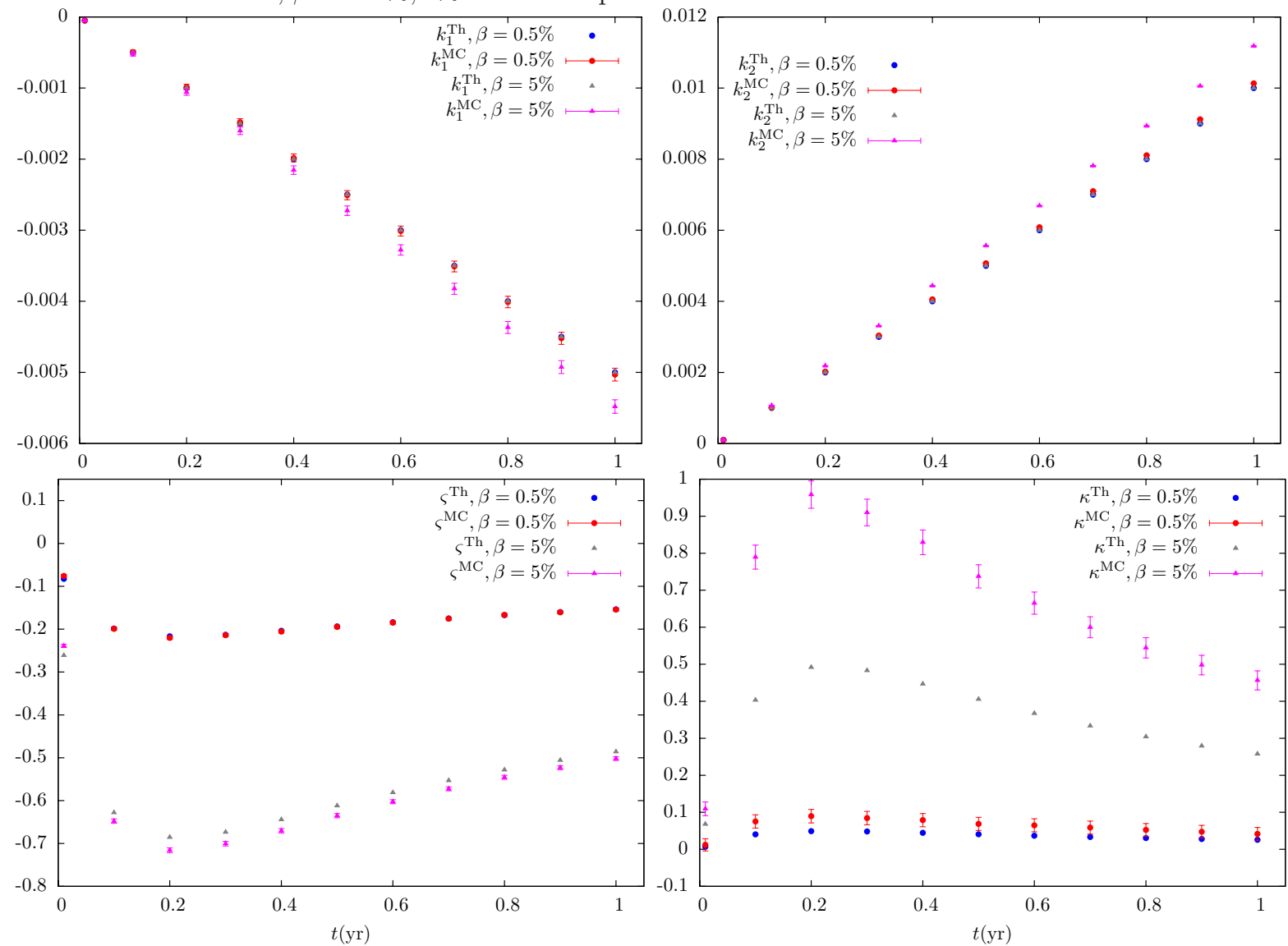

Table 1. Scaling of normalized cumulants for increasing values of $\beta$. The time horizon is given by $t-t_{0}=1$, while the other parameters are the same of Fig. 1 The index MC refers to values numerically computed with MC simulation (between parenthesis we report the error on the last significant digit, $95 \%$ confidence level), while cumulants with index ${ }^{\text {Th }}$ correspond to Eq. (17)-Eq. (20).

\begin{tabular}{llllllll}
\hline$\beta(\%)$ & 0.5 & 1 & 2 & 5 & 10 & 25 & 50 \\
\hline$k_{1}^{\mathrm{MC}}$ & $-0.00503(8)$ & $-0.00508(8)$ & $-0.00518(8)$ & $-0.0055(1)$ & $-0.0060(1)$ & $-0.0080(1)$ & $-0.0131(2)$ \\
$k_{1}^{\mathrm{Th}}$ & -0.005 & -0.005 & -0.005 & -0.005 & -0.005 & -0.005 & -0.005 \\
\hline$k_{2}^{\mathrm{MC}}$ & $0.01013(1)$ & $0.01025(1)$ & $0.01048(1)$ & $0.01118(2)$ & $0.01242(2)$ & $0.01702(4)$ & $0.02932(8)$ \\
$k_{2}^{\mathrm{Th}}$ & 0.01 & 0.01 & 0.01 & 0.01 & 0.01 & 0.01 & 0.01 \\
\hline$\varsigma^{\mathrm{MC}}$ & $-0.154(4)$ & $-0.219(4)$ & $-0.311(4)$ & $-0.502(4)$ & $-0.733(6)$ & $-1.29(1)$ & $-2.22(2)$ \\
$\varsigma^{\mathrm{Th}}$ & -0.154 & -0.217 & -0.307 & -0.486 & -0.687 & -1.087 & -1.54 \\
\hline$\kappa^{\mathrm{MC}}$ & $0.04(2)$ & $0.08(2)$ & $0.17(2)$ & $0.46(2)$ & $0.99(4)$ & $3.2(1)$ & $10.3(6)$ \\
$\kappa^{\mathrm{Th}}$ & 0.026 & 0.0515 & 0.10 & 0.26 & 0.51 & 1.29 & 2.6 \\
\hline
\end{tabular}




\section{Limit case II: expansion for small $\beta$}

The numerical results shown in Section 3 suggest that the level of the stationary variance of $Y$ is a crucial parameter to describe accurately the returns distribution and for this reason we focus on it. The intuition behind our choice is that, since $\beta$ governs the level of the fluctuations of $Y$ around its stationary value, keeping $\beta$ low allows us to linearize the exponential form of the volatility. Moreover we will show in the next subsection how it is possible to exactly solve the Fokker-Planck equation associated with the system of SDEs by means of linearization. We limit our investigation to $0<\beta \leq 0.1$. Higher values can be explored and the goodness of the approximation can be tested comparing the numerical results obtained via MC simulation of the linear and complete dynamics. However, it is important to note that in practical applications it is usually required to keep the volatility non negative. The probability for the volatility to become negative can be easily computed and is given by the following formula

$$
\frac{1}{2} \operatorname{Erfc}\left(\frac{1+\gamma+\left(y_{0}-\gamma\right) \mathrm{e}^{-\alpha\left(t-t_{0}\right)}}{\sqrt{\beta\left(\mathrm{e}^{-2 \alpha\left(t-t_{0}\right)}+1\right)}}\right) \stackrel{t-t_{0} \gg 1 / \alpha}{\longrightarrow} \frac{1}{2} \operatorname{Erfc}\left(\frac{1+\gamma}{\sqrt{\beta}}\right),
$$

where Erfc is the complementary error function. After few relaxation times $1 / \alpha$ and

for $\gamma$ of order 0 we have that the probability reduces to one half of $\operatorname{Erfc}(\sqrt{2 \alpha} / \mathrm{k})$. For $\beta=1 \%$ we have a probability of order $10^{-45}$, while for $\beta=10 \%$ it increases to $4 \times 10^{-6}$, which is still a very small value.

\subsection{Exact solution for the linear model}

The starting point of our analysis is the linearization of Eq. (5). Since when the $Y$ process termalizes the random variable fluctuates around $\gamma$, it is quite natural to expand the exponential around the stationary value. By defining $\bar{m} \doteq m \mathrm{e}^{\gamma}$ and introducing the random variable $Z \doteq Y-\gamma+1$, Eq. (51) and Eq. (21) can be rewritten as

$$
\begin{aligned}
& \mathrm{d} X=-\frac{\bar{m}^{2}}{2}(2 Z-1) \mathrm{d} t+\bar{m} Z \mathrm{~d} W_{1}, \\
& X\left(t_{0}\right)=0, \\
& \mathrm{~d} Z=\alpha(1-Z) \mathrm{d} t+k \rho \mathrm{d} W_{1}(t)+k \sqrt{1-\rho^{2}} \mathrm{~d} W_{2}(t), \\
& Z\left(t_{0}\right)=y_{0}-\gamma+1,
\end{aligned}
$$

To derive the analytical expression of $p_{X}\left(X(t) \mid X_{0}, Z_{0}\right)$ we will follow a strategy analogous to the one pioneered by Heston in [13]. For notational convenience we indicate $p_{X}\left(x \mid x_{0}, z_{0}\right)$ shortly as $p_{X}$. The Fokker-Planck backward equation satisfied by $p_{X}$ is readily written as

$$
\begin{aligned}
\frac{\partial}{\partial t_{0}} p_{X} & =\frac{\bar{m}^{2}}{2}\left(2 z_{0}-1\right) \frac{\partial}{\partial x_{0}} p_{X}-\alpha\left(1-z_{0}\right) \frac{\partial}{\partial z_{0}} p_{X} \\
& -\frac{\bar{m}^{2}}{2} z_{0}^{2} \frac{\partial^{2}}{\partial x_{0}^{2}} p_{X}-\rho k \bar{m} z_{0} \frac{\partial^{2}}{\partial x_{0} \partial z_{0}} p_{X}-\frac{k^{2}}{2} \frac{\partial^{2}}{\partial z_{0}^{2}} p_{X}
\end{aligned}
$$


Heston technique essentially reduces to the observation that, if we assume as a final time condition the expression $\mathrm{e}^{i x \phi}$, Eq. (27) is precisely the partial differential equation governing the evolution of the characteristic function $f\left(\phi ; x_{0}, z_{0}\right)$ implicitly defined by

$$
p_{X}\left(x \mid x_{0}, z_{0}\right)=\frac{1}{2 \pi} \int_{-\infty}^{+\infty} \mathrm{e}^{-i \phi x} f\left(\phi ; x_{0}, z_{0}\right) \mathrm{d} \phi .
$$

We try a solution of the form

$$
f\left(\phi ; x_{0}, z_{0}\right)=\mathrm{e}^{\mathcal{A}\left(t-t_{0}, \phi\right)+\mathcal{B}\left(t-t_{0}, \phi\right) z_{0}+\mathcal{C}\left(t-t_{0}, \phi\right) z_{0}^{2}+i \phi x_{0}} .
$$

We substitute it in Eq. (27) and we get

$$
\begin{aligned}
\dot{\mathcal{A}}+\dot{\mathcal{B}} z_{0}+\dot{\mathcal{C}} z_{0}^{2} & =\frac{\bar{m}^{2}}{2}\left(2 z_{0}-1\right) i \phi-\frac{\bar{m}^{2}}{2} z_{0}^{2}\left(-\phi^{2}\right)-\alpha\left(1-z_{0}\right)\left(\mathcal{B}+2 \mathcal{C} z_{0}\right) \\
& -\frac{k^{2}}{2}\left[\left(\mathcal{B}+2 \mathcal{C} z_{0}\right)^{2}+2 \mathcal{C}\right]-\rho k \bar{m} z_{0} i \phi\left(\mathcal{B}+2 \mathcal{C} z_{0}\right)
\end{aligned}
$$

where the dot stays for a derivative w.r.t. $t_{0}$. If we collect the quadratic and linear terms, independent of $z_{0}$, we have the ODEs satisfied by $\mathcal{C}, \mathcal{B}$ and $\mathcal{A}$ respectively:

$$
\begin{aligned}
\dot{\mathcal{C}} & =\frac{\bar{m}^{2}}{2} \phi^{2}+2 \alpha \mathcal{C}-2 k^{2} \mathcal{C}^{2}-\rho k \bar{m} i \phi 2 \mathcal{C}, \\
\dot{\mathcal{B}} & =\bar{m}^{2} i \phi-2 \alpha \mathcal{C}+\alpha \mathcal{B}-2 k^{2} \mathcal{B C}-\rho k \bar{m} i \phi \mathcal{B}, \\
\dot{\mathcal{A}} & =-\frac{\bar{m}^{2}}{2} i \phi-\alpha \mathcal{B}-\frac{k^{2}}{2}\left(\mathcal{B}^{2}+2 \mathcal{C}\right),
\end{aligned}
$$

with final time conditions

$$
\begin{aligned}
& \mathcal{C}(0, \phi)=0, \\
& \mathcal{B}(0, \phi)=0, \\
& \mathcal{A}(0, \phi)=0 .
\end{aligned}
$$

Equation (31) is a Riccati type ODE and once it has been solved, we insert the solution in Eq. (32) and Eq. (33) and we integrate them out in the usual way. The explicit expression for $\mathcal{C}, \mathcal{B}$ and $\mathcal{A}$ are quite involved. To improve the readability we define some auxiliary variables

$$
\begin{aligned}
& d \doteq 2 \sqrt{k^{2} \bar{m}^{2} \phi^{2}+(\alpha-i k \bar{m} \rho \phi)^{2}}, \quad b \doteq 2(\alpha-i k \bar{m} \rho \phi), \\
& g \doteq \frac{b-d}{b+d}, \quad h \doteq i \bar{m}^{2} \phi \quad \text { and } \quad n \doteq \frac{\alpha}{2 k^{2}}(b-d) \text {. }
\end{aligned}
$$

Now the desired functions read

$$
\begin{aligned}
& \mathcal{C}\left(t-t_{0}, \phi\right)=\frac{b-d}{4 k^{2}} \frac{1-\mathrm{e}^{-d\left(t-t_{0}\right)}}{1-g \mathrm{e}^{-d\left(t-t_{0}\right)}} \\
& \mathcal{B}\left(t-t_{0}, \phi\right)=2 \frac{\mathrm{e}^{-\frac{1}{2} d\left(t-t_{0}\right)}((g+1) h-2 n)+n+\mathrm{e}^{-d\left(t-t_{0}\right)}(n-g h)-h}{d\left(1-g \mathrm{e}^{-d\left(t-t_{0}\right)}\right)}
\end{aligned}
$$


and

$$
\begin{aligned}
& \mathcal{A}\left(t-t_{0}, \phi\right)=\frac{1}{2} h\left(t-t_{0}\right) \\
& +2 \alpha\left\{\frac { ( g + 1 ) h - 2 n } { d ^ { 2 } \sqrt { g } } \left[\ln \left(1-\sqrt{g} \mathrm{e}^{-\frac{d}{2}\left(t-t_{0}\right)}\right)-\ln (1-\sqrt{g})\right.\right. \\
& \left.-\ln \left(1+\sqrt{g} \mathrm{e}^{-\frac{d}{2}\left(t-t_{0}\right)}\right)+\ln (1+\sqrt{g})\right] \\
& +\frac{n(g+1)-2 g h}{d^{2} g}\left[\ln \left(1-g \mathrm{e}^{-d\left(t-t_{0}\right)}\right)-\ln (1-g)\right] \\
& \left.+\frac{n-h}{d}\left(t-t_{0}\right)\right\} \\
& +2 k^{2}\left\{-\frac{(n-g h)^{2}}{d^{3} g}\left[\frac{\mathrm{e}^{-d\left(t-t_{0}\right)}-1}{(1-g)\left(1-g \mathrm{e}^{-d\left(t-t_{0}\right)}\right)}+\frac{1}{g}\left(\ln \left(1-g \mathrm{e}^{-d\left(t-t_{0}\right)}\right)\right.\right.\right. \\
& -\ln (1-g))] \\
& -\frac{((g+1) h-2 n)^{2}+2(n-g h)(n-h)}{d^{3}} \times \frac{\mathrm{e}^{-d\left(t-t_{0}\right)}-1}{(1-g)\left(1-g \mathrm{e}^{-d\left(t-t_{0}\right)}\right)} \\
& +\frac{(n-h)^{2}}{d^{2}}\left[\left(t-t_{0}\right)+\frac{1}{d}\left(\ln \left(1-g \mathrm{e}^{-d\left(t-t_{0}\right)}\right)-\ln (1-g)\right)\right. \\
& \left.-\frac{g}{d} \frac{\mathrm{e}^{-d\left(t-t_{0}\right)}-1}{(1-g)\left(1-g \mathrm{e}^{-d\left(t-t_{0}\right)}\right)}\right] \\
& +\left[\frac{((g+1) h-2 n)^{2}}{d^{3} g \sqrt{g}}-\frac{((g+1) h-2 n)(n-h)}{d^{3} \sqrt{g}}\right] \\
& \times\left[\ln \left(1+\sqrt{g} \mathrm{e}^{-\frac{d}{2}\left(t-t_{0}\right)}\right)-\ln (1+\sqrt{g})-\ln \left(1-\sqrt{g} \mathrm{e}^{-\frac{d}{2}\left(t-t_{0}\right)}\right)\right. \\
& +\ln (1-\sqrt{g})] \\
& -2\left[\frac{((g+1) h-2 n)^{2}}{d^{3} g}+\frac{((g+1) h-2 n)(n-h)}{d^{3}}\right] \\
& \left.\times \frac{\left(1+g \mathrm{e}^{-\frac{d}{2}\left(t-t_{0}\right)}\right)\left(\mathrm{e}^{-\frac{d}{2}\left(t-t_{0}\right)}-1\right)}{(1-g)\left(1-g \mathrm{e}^{-d\left(t-t_{0}\right)}\right)}\right\} \\
& +\frac{b-d}{4}\left\{\left(t-t_{0}\right)+\frac{g-1}{d g}\left[\ln \left(1-g \mathrm{e}^{-d\left(t-t_{0}\right)}\right)-\ln (1-g)\right]\right\}
\end{aligned}
$$

The three functions $\mathcal{A}, \mathcal{B}$ and $\mathcal{C}$ require some care. Indeed, similarly to the Heston case, non trivial problems emerge due to the multi-valued nature of complex square root and logarithm. In particular, due to "branching" effects, the characteristic function can become discontinuous. Following the same arguments discussed in [39, 40, 41], we checked the smoothness of $f\left(\phi ; x_{0}, z_{0}\right)$ for the set of parameters used in the next Section.

The complete characterization of $f\left(\phi ; x_{0}, z_{0}\right)$ is the main result of this paper. The only remaining task to be performed to obtain the probability distribution in the direct space is to Fourier anti-transform it. We stress again that the obtained solution is an exact one but for the linear model. In the following Section we provide a set of $\beta$ values for which we have numerically tested the effectiveness of the approximation. 


\subsection{Further Numerical Results}

The exact solution for the probability distribution of returns in the framework of the linear problem is given in an implicit way by means of a Fourier anti-transformation of Eq. (28), which can not be computed analytically. We compared two different ways for the numerical evaluation of $p_{X}$ : the integration with a trapezoidal approximation and the FFT algorithm. In order to improve the efficiency of the methods one can observe that $p_{X}$ is a real function, so the integrand of Eq. (28) must have its real part even and its imaginary part odd. We can integrate on one half of the entire real axis and take twice the real part of the result. To sum up:

$$
p_{X}\left(x \mid x_{0}, z_{0}\right)=\frac{1}{\pi} \operatorname{Re}\left[\int_{0}^{+\infty} \mathrm{e}^{-i \phi x} f\left(\phi ; x_{0}, z_{0}\right) \mathrm{d} \phi\right] .
$$

We checked the convergence of the two methods mentioned previously increasing the number of points for the sampling of the integrand. All the results presented in the next figures have been obtained with the FFT algorithm with $2^{22}$ points on the fixed interval of integration $\left[0,10^{3}\right]$. The tails of the resulting distribution must decrease to zero, but they are extremely sensitive to the FFT frequency sampling so they have been neglected in Fig. 4. For the reasons explained in Section 3.2, we chose $\Delta t=10^{-4}$ and $\mathrm{MCPATHS}=5 \cdot 10^{6}$ to simulate the dynamics of the exponential and linear models.

In Fig. 4 we report the histograms of returns obtained via MC simulations of the exponential and the linear models with the $p_{X}$ curve given by the numerical Fourier anti-transformation. $m=0.1, \alpha=10, \gamma=0$ and $y_{0}=0$ are fixed for all the figures and table of this Section. Each group of three curves corresponds to $\beta$ values $0.5 \%$, $1 \%, 2 \%, 5 \%$ and 10\%, from bottom to top respectively. Again each curve has been shifted for the sake of readability. The top frame has been obtained with a positive $\rho$ value (0.5), which is evident from the rightward asymmetry of the curves, while, in the bottom panel, curves have large leftward asymmetry corresponding to $\rho=-0.9$. The agreement between theory prediction and $\mathrm{MC}$ simulation is very good for $\beta=0.5 \%$, $1 \%$ and $2 \%$, in both cases of $\rho$ values. The distributions of returns from exponential model are well reproduced by the ones from linear model and, as a consequence, by the semi-analytical approximation $p_{X}$. The agreement worsen when $\beta$ increases to $5 \%$ and $10 \%$, as it can be seen observing the fatter tail in each panel. All the curves in Fig. 4s show that the returns distribution in the linear model is exactly the one computed via FFT, thus confirming the goodness of the exact solution. Finally we observe the good behavior of all the semi-analytical curves, which, since they are exact solutions of a Fokker-Planck equation, do not become negative or fluctuate like in the case of the Edgeworth expansion considered in the previous Section.

Again, to make the considerations more quantitative, in Fig. 5 the scaling with time of mean, variance, skewness and kurtosis is shown for $\beta=0.5 \%$ and $5 \%, \rho=-0.9$. Then in Tab. 2, the numerical values of normalized cumulants computed with MC samples of returns from exponential and linear models are shown for $\beta=1 \%, 2 \%$ and $t-t_{0}=0.01,0.1,0.2,0.5,1$. The results show that, for $\beta \leq 2 \%$, mean, skewness 
Figure 4. Returns distributions from MC simulation of exponential and linear models in comparison with the probability distributions given by Eq. (28), numerically computed by FFT algorithm. Parameters values as discussed in the text, curves from bottom to top correspond to increasing values of $\beta$ : $0.5 \%, 1 \%, 2 \%, 5 \%$ and $10 \%$. Curves have been shifted upwards. Top panel: $\rho=0.5$ and $t-t_{0}=1$; bottom panel: $\rho=-0.9$ and $t-t_{0}=1$.
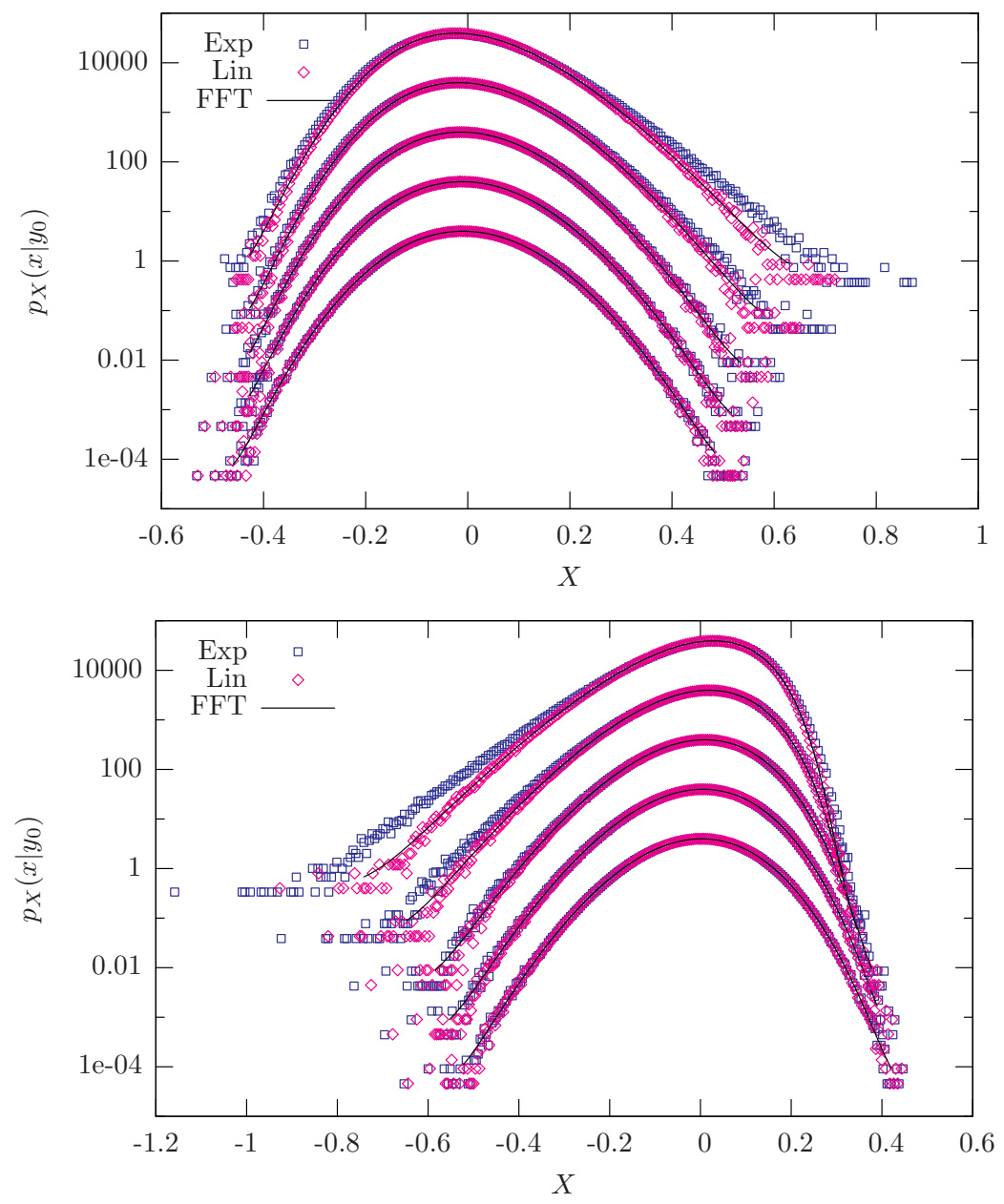

and kurtosis from the complete and linear models agree within the usual 95\% MC confidence level, while variance values are not statistically compatible. This is an expected result, since the linear model can not exactly reproduce the complete one and for this reason we also expect that for higher values of MCPATHS the discrepancies for the other cumulants should emerge. However, we are able to evaluate the relative differences among cumulants values and we can evaluate the degree of approximation of the analytical solution: for $\beta=1 \%$ the relative disagreement between $k_{2}^{\text {Exp }}$ and $k_{2}^{\text {Lin }}$ increases with time from $0.6 \%$ to $1 \%$. As expected, the disagreement between cumulants computed for the exponential and linear models increases with the value of $\beta$.

In comparison with the results given in Section 3.2, one can note that the tails of the distribution are better reproduced by the semi-analytical approximation (for mentioned 
Figure 5. From top left clockwise: scaling with time of mean, variance, kurtosis and skewness. Comparison between numerically estimated values of normalized cumulants for the exponential (Eq. (2), Eq. (5)) and the linear models (with MC 95\% confidence level) . $\rho=-0.9, \beta=0.5 \%, 5 \%$ and other parameters as in the text.
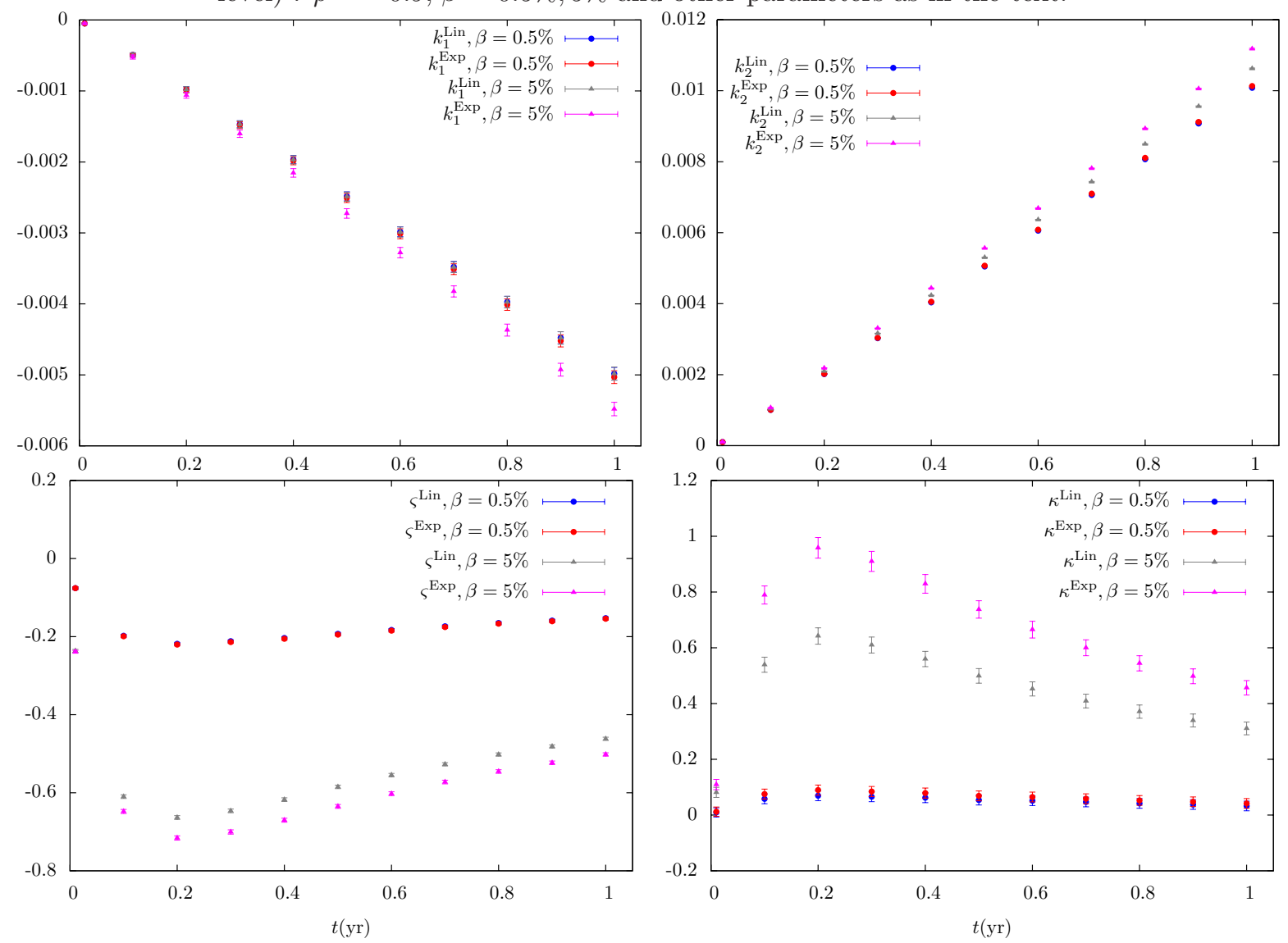

values of $\beta$ ) than by the Edgeworth expansion, the other parameters being fixed. Also the general agreement of cumulants for $\beta \leq 2 \%$ is better than the corresponding results given in Fig. 3 and Tab. 1 .

\section{Real data analysis}

We test the effectiveness of previous analytical results on a data set composed by several financial indexes. In particular we consider the following indexes from the equity sector, DAX30, CAC40, FTSE100, S\&PMib, S\&P500, DJ Euro Stoxx 50 and NYSE, but we detail the analysis only for DAX30 and DJ Euro Stoxx 50. Similar results have been found also for the other series.

DAX30 time series is made of 12173 daily close prices, from $4^{\text {th }}$ January 1960 until $30^{\text {th }}$ June 2008, while for DJ Euro Stoxx 50 we consider 5566 prices, from $1^{\text {st }}$ January 1987 until $31^{\text {st }}$ August 2008. Time is measured on a yearly base, so for DAX30 and DJ Euro Stoxx 50 we have 48.5 years and 21.75 years time windows respectively. The model described by Eqs. (11) and (2) depends on 8 parameters. However, $s_{0}$ corresponds 
Table 2. Scaling with time of normalized cumulants for $\beta=1 \%$ and $2 \%, \rho=-0.9$ and other parameters as in the text. The indexes Exp and Lin refer to values numerically computed with MC simulations with exponential and linear dynamics (between parenthesis we report the error on the last significant digit, $95 \%$ confidence level).

\begin{tabular}{|c|c|c|c|c|c|}
\hline \multirow[b]{2}{*}{$t-t_{0}(\mathrm{yr})$} & \multicolumn{5}{|c|}{$\beta=1 \%$} \\
\hline & 0.01 & 0.1 & 0.2 & 0.5 & 1 \\
\hline$k_{1}^{\operatorname{Exp}}\left(10^{-4}\right)$ & $-0.50(8)$ & $-6.0(2)$ & $-9.9(4)$ & $-25.3(6)$ & $-50.8(9)$ \\
\hline$k_{1}^{\operatorname{Lin}}\left(10^{-4}\right)$ & $-0.50(8)$ & $-4.9(2)$ & $-9.8(4)$ & $-24.9(6)$ & $-49.8(9)$ \\
\hline$k_{2}^{\operatorname{Exp}}\left(10^{-4}\right)$ & $1.002(1)$ & $10.15(1)$ & $20.42(2)$ & $51.26(6)$ & $102.5(1)$ \\
\hline$k_{2}^{\operatorname{Lin}}\left(10^{-4}\right)$ & $1.001(1)$ & $10.09(1)$ & $20.26(2)$ & $50.79(6)$ & $101.5(1)$ \\
\hline$\varsigma^{\operatorname{Exp}}$ & $-0.107(3)$ & $-0.282(4)$ & $-0.312(4)$ & $-0.276(4)$ & $-0.219(4)$ \\
\hline$\varsigma^{\operatorname{Lin}}$ & $-0.107(3)$ & $-0.279(4)$ & $-0.306(4)$ & $-0.271(4)$ & $-0.215(4)$ \\
\hline$\kappa^{\operatorname{Exp}}$ & $0.02(1)$ & $0.15(2)$ & $0.18(2)$ & $0.14(2)$ & $0.08(2)$ \\
\hline \multirow[t]{2}{*}{$\kappa^{\operatorname{Lin}}$} & $0.02(2)$ & $0.11(2)$ & $0.14(2)$ & $0.11(2)$ & $0.07(2)$ \\
\hline & \multicolumn{5}{|c|}{$\beta=2 \%$} \\
\hline$k_{1}^{\operatorname{Exp}}\left(10^{-4}\right)$ & $-0.50(8)$ & $-5.0(2)$ & $-10.1(4)$ & $-25.8(6)$ & $-51.8(9)$ \\
\hline$k_{1}^{\operatorname{Lin}}\left(10^{-4}\right)$ & $-0.50(8)$ & $-4.9(2)$ & $-9.8(4)$ & $-24.9(6)$ & $-49.8(9)$ \\
\hline$k_{2}^{\operatorname{Exp}}\left(10^{-4}\right)$ & $1.004(1)$ & $10.28(1)$ & $20.77(2)$ & $52.35(7)$ & $104.8(1)$ \\
\hline$k_{2}^{\operatorname{Lin}}\left(10^{-4}\right)$ & $1.002(1)$ & $10.16(1)$ & $20.45(2)$ & $51.38(6)$ & $102.8(1)$ \\
\hline$\varsigma^{\operatorname{Exp}}$ & $-0.151(4)$ & $-0.402(4)$ & $-0.443(4)$ & $-0.393(4)$ & $-0.311(4)$ \\
\hline$\varsigma^{\operatorname{Lin}}$ & $-0.150(4)$ & $-0.392(4)$ & $-0.429(4)$ & $-0.380(2)$ & $-0.300(4)$ \\
\hline$\kappa^{\operatorname{Exp}}$ & $0.04(2)$ & $0.30(2)$ & $0.36(2)$ & $0.28(2)$ & $0.17(2)$ \\
\hline$\kappa^{\operatorname{Lin}}$ & $0.03(2)$ & $0.22(2)$ & $0.27(2)$ & $0.21(2)$ & $0.13(2)$ \\
\hline
\end{tabular}

to the asset spot price so the true free parameters are $\mu, m, y_{0}, \alpha, \gamma, \beta$ and $\rho$. In order to estimate their values we adopt the following strategies:

$\mu$ : from the discretized version of equation (1)

$$
\frac{\Delta S_{i}}{S_{i}}=\mu \Delta t+m \sqrt{\Delta t} \mathrm{e}^{Y_{i}} \epsilon_{i}
$$

where $\Delta S_{i}=S_{i+1}-S_{i}$ and $\epsilon_{i} \sim \mathcal{N}(0,1)$, we can conclude that the expectation $\left\langle\Delta S_{i} / S_{i}\right\rangle / \Delta t$ over the real data sample provides an estimate of $\mu$. For DAX30 $\Delta t=3.98 \times 10^{-3}$, while for DJ Euro Stoxx 50 we have $\Delta t=3.91 \times 10^{-3}$.

$\gamma, y_{0}$ : Remembering the definition $\sigma(t)=m \mathrm{e}^{Y(t)}$, it is readily proved that

$$
\mathbb{E}\left[\sigma(t)^{n}\right]=m^{n} \mathrm{e}^{n \mathbb{E}[Y(t)]} \mathrm{e}^{\frac{n^{2}}{2}\left(\mathbb{E}\left[Y(t)^{2}\right]-\mathbb{E}[Y(t)]^{2}\right),}
$$

with $\mathbb{E}[Y(t)]$ and $\mathbb{E}\left[Y(t)^{2}\right]$ given in Eqs. (3) and (4). It is common practice to assume that, for the observed time series, the volatility process has already reached the stationary state. Under this assumption, previous expression reduces 
to $\mathbb{E}\left[\sigma(t)^{n}\right]=\left(m \mathrm{e}^{\gamma}\right)^{n} \mathrm{e}^{\frac{n^{2}}{2} \beta}$. It is crucial noticing that all the moments do not depend on $y_{0}$ and $\mathrm{e}^{\gamma}$ is always coupled with $m$. For this reason we introduce the parameter $\bar{m}=m \mathrm{e}^{\gamma}$ (see also lines before Eq.(50) ) and we set $y_{0}$ equal to zero.

$\bar{m}, \beta$ : These two parameters completely specify the stationary log-normal distribution of the stochastic variable $\sigma$. To extract the distribution of the hidden variable $\sigma$ from the series of financial returns, we implement the methodology described in Appendix B of reference [42]. The consistency of our code has been tested over the NYSE time series and we have found results in full agreement with those quoted in 42 .

$\alpha, \rho$ : Finally, to estimate $\alpha$ and $\rho$, we search for values able to reproduce the empirical scaling with time of real data skewness and kurtosis. We consider time horizons from one day to one hundred days and normalized cumulants are evaluated with standard estimators. By means of centered returns, computed from market prices, we obtain the empirical skewness, $\varsigma_{P h}$, and kurtosis, $\kappa_{P h}$, and corresponding errors, $\epsilon_{P h}^{\varsigma}$ and $\epsilon_{P h}^{\kappa}$. By generating 10000 paths from the exponential Ornstein-Uhlenbeck dynamics, we can compute the $\mathrm{MC}$ estimators $\varsigma_{M C}, \kappa_{M C}$ and associated errors, $\epsilon_{M C}^{\varsigma}$ and $\epsilon_{M C}^{\kappa}$. The optimal $\alpha$ and $\rho$ are given by those values that minimize the sum over 100 time horizons of the normalized squared differences, according to the following formula:

$$
\left(\alpha^{*}, \rho^{*}\right)=\min _{\alpha>0, \rho \in(-1,1)} \sum_{i=1}^{100}\left[\frac{\left(\varsigma_{i, P h}-\varsigma_{i, M C}\right)^{2}}{\epsilon_{i, P h}^{\varsigma}+\epsilon_{i, M C}^{\varsigma}}+\frac{\left(\kappa_{i, P h}-\kappa_{i, M C}\right)^{2}}{\epsilon_{i, P h}^{\kappa}{ }^{2}+\epsilon_{i, M C}^{\kappa}}\right] .
$$

The subscript $i$ means that the corresponding quantity is evaluated at time $i \Delta t$. Since the presence of noisy denominators in Eq. (43), we can not resort to optimization algorithms based on gradient methods. For this reason we implement the principal axis approach with the one dimensional search based on the Brent method (see opt/ directory at http://www.netlib.org).

The probability distribution $P(\sigma)$ of volatility is plotted in Fig. 6, both for the DAX30 index and the DJ Euro Stoxx 50. Distributions tails suffer low statistics effects, but in the central region they are well fitted by a log-normal distribution

$$
p(\sigma)=\frac{1}{\sqrt{2 \pi} s \sigma} \exp -\frac{1}{2}\left(\frac{\log \sigma-\log \sigma_{0}}{s}\right)^{2} .
$$

The fit is performed in the range $0.0004 \leq \sigma \leq 0.015$ for DAX30 and gives $\log \sigma_{0}=$ $-4.492 \pm 0.001$ and $s=0.334 \pm 0.001$, while for the DJ Euro Stoxx 50 the log-normal fit is consistent in a wider region $0.0015 \leq \sigma \leq 0.015$ and gives $\log \sigma_{0}=-4.7049 \pm 0.0008$ and $s=0.5147 \pm 0.0007$. Tab. 3 details the corresponding values for $\bar{m}=\sigma_{0} / \sqrt{\Delta t}$ and $\beta=s^{2}$. In Fig. 7 we plot the scaling with time of skewness and kurtosis for the DJ Euro Stoxx 50 index. We have found similar results for DAX30, but we do not report them here. Error bars represent the $68 \%$ confidence level (CL), while the solid lines correspond to the analytical expressions given by Eqs. (19) and (20). These lines have been generated using parameters values obtained by the minimization of r.h.s. of 
Figure 6. Probability distribution $P(\sigma)$ of volatility for DAX30 (blue boxes) and DJ Euro Stoxx 50 (violet diamonds). Solid lines correspond to a log-normal fit.

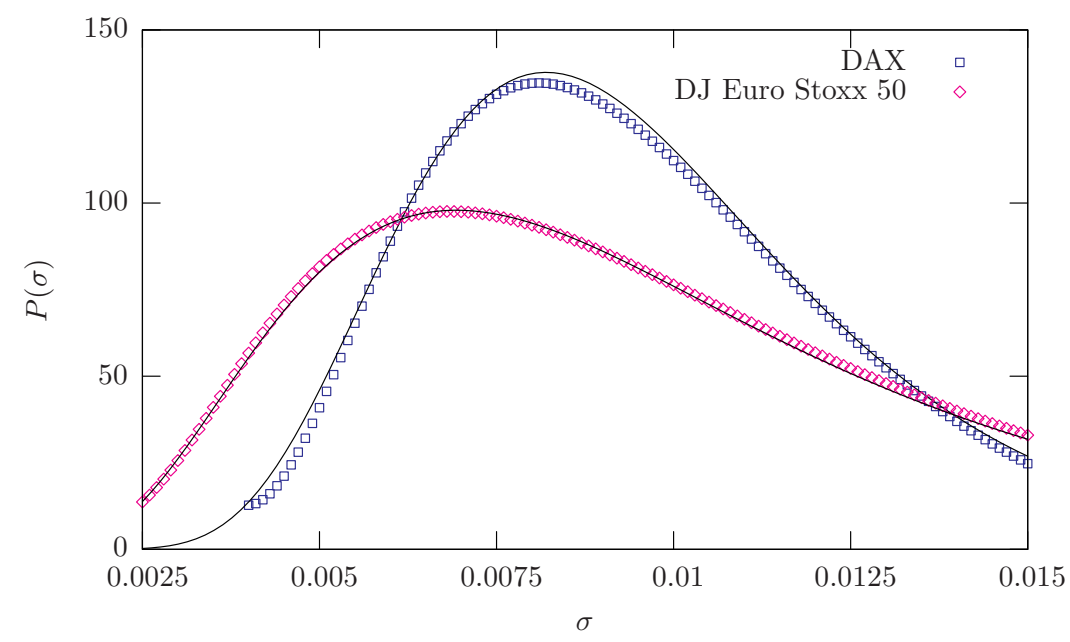

Table 3. Estimated values for the model parameters for DAX30 and DJ Euro Stoxx 50 indexes.

\begin{tabular}{llllllll}
\hline & $\mu\left(\mathrm{yr}^{-1}\right)$ & $y_{0}$ & $\gamma$ & $\bar{m}\left(\mathrm{yr}^{-\frac{1}{2}}\right)$ & $\beta$ & $\alpha\left(\mathrm{yr}^{-1}\right)$ & $\rho$ \\
\hline DAX30 & $7.39 \times 10^{-2}$ & 0 & 0 & $14.52 \times 10^{-2}$ & $11.16 \times 10^{-2}$ & 30.76 & -0.54 \\
\hline DJ Euro Stoxx 50 & $7.97 \times 10^{-2}$ & 0 & 0 & $14.45 \times 10^{-2}$ & $26.51 \times 10^{-2}$ & 47.74 & -0.52 \\
\hline
\end{tabular}

Figure 7. Scaling with time of skewness (left panel) and kurtosis (right panel) for DJ Euro Stoxx 50 index. We plot the scaling of empirical cumulants (violet crossed-line) with the corresponding $68 \% \mathrm{CL}$ bars and cumulants with $68 \% \mathrm{CL}$ bars from the MC simulation (blue boxed-line). The solid line corresponds to the analytical expressions given in Eqs. (19) and (20).

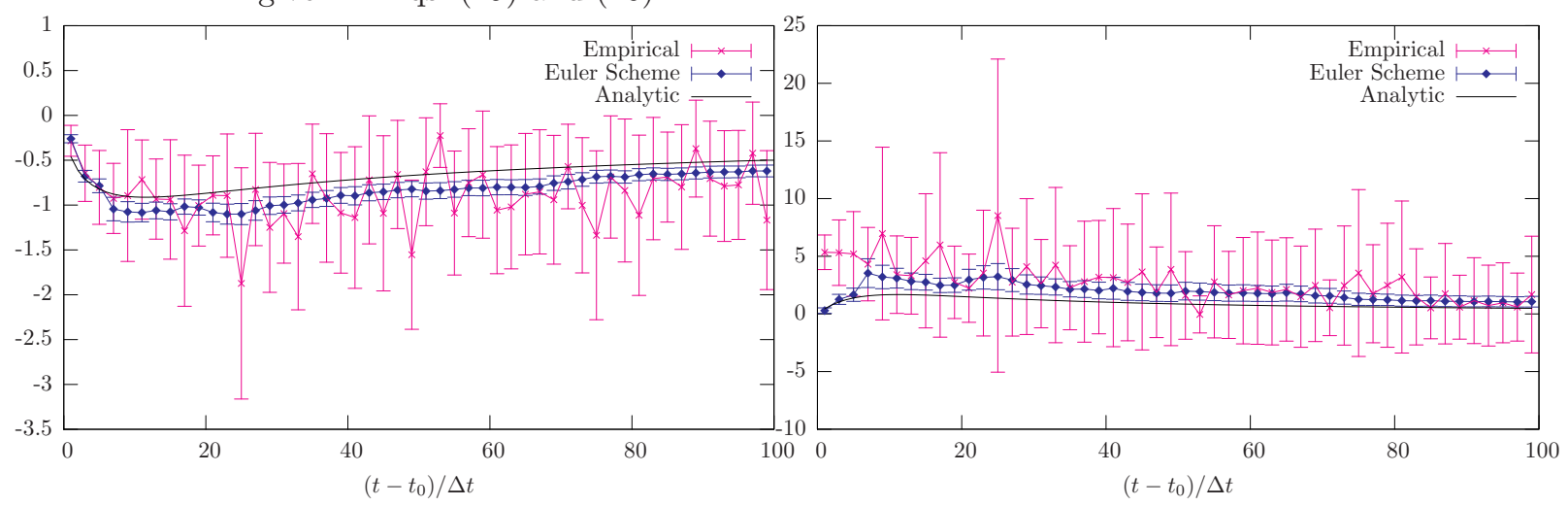

Eq. (43). Their values are detailed in Tab. 3. It is worth to comment that in order to capture the leftward asymmetry of the real data distribution the correlation coefficient $\rho$ is correctly predicted to be negative. The relaxation time $1 / \alpha \sim 5$ days implies a quite fast thermalization process. The plotted results indicate that the exponential 
Ornstein-Uhlenbeck model is unable to capture the excess of kurtosis observed in many high-frequency returns distributions [18, 43], but it can provide reliable predictions for returns distributions corresponding to sufficiently long-time lags and is able to account for the transition from a leptokurtic to a Gaussian regime, as observed in other SV models [20, 21]. Finally, we present the comparison between the probability distributions

Figure 8. DAX30 index returns distributions for two different time horizons, 25 trading days (first row) and 45 trading days (second row).
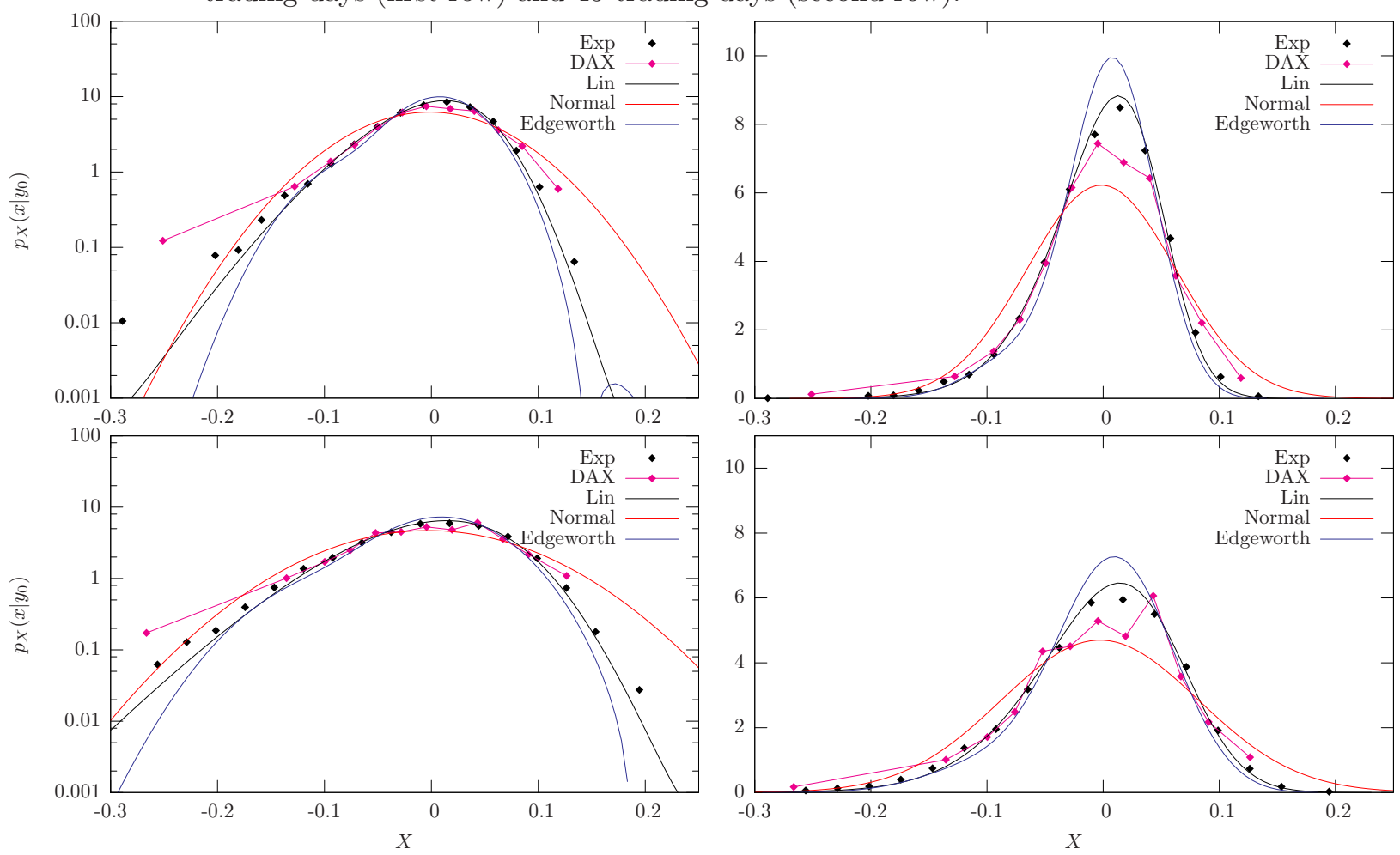

for DAX30, see Fig. 8, and for DJ Euro Stoxx 50, see Fig. 9, computed according to the various models we have discussed in this work. First row of both figures is obtained setting the time horizon equal to 25 trading days, while the second one corresponds to the 45 trading days horizon. The distributions are presented both in log-linear and linear scales, in order to allow the reader to appreciate the behavior on the tails and in the central region, respectively. The boxed-lines represent the empirical histograms (all the bins contain at least ten points), while black points correspond to the histograms from the MC simulation of the exponential Ornstein-Uhlenbeck model. Moreover, we report the Fourier transform of the characteristic function for the linear model (solid black line), the Normal Maximum Likelihood fit (solid red line) and the analytical solution (16) (solid blue line). The Normal approximation is scarcely representative of the true empirical distribution, while the exponential model captures in a quite effective way the leftward asymmetry, the fatter tails and the narrower central region. The Fourier transform of Eq. (29) and Eq. (16) are both candidates to approximate the distribution of the exponential model. For the DAX30 index, the level of $\beta$ equal to $11.16 \%$ allow us to be confident in a good performance of the linear model. This is indeed the case, 
as it is clearly shown in Fig. 8. The situation slightly worsen for the DJ Euro Stoxx 50 index, where $\beta=26.51 \%$. However, also in this case, the linear model performs better than the solution based on the Edgeworth expansion, that presents a narrower central peak and thinner tails. Moreover, for the r.h.s. of Eq. (16) positive definiteness is not guaranteed. This is confirmed looking at the right tails in Fig. 8, where the distributions in log-linear scale have to be truncated since the presence of negative values.

Figure 9. DJ Euro Stoxx 50 index returns distributions two different time horizons, 25 trading days (first row) and 45 trading days (second row).
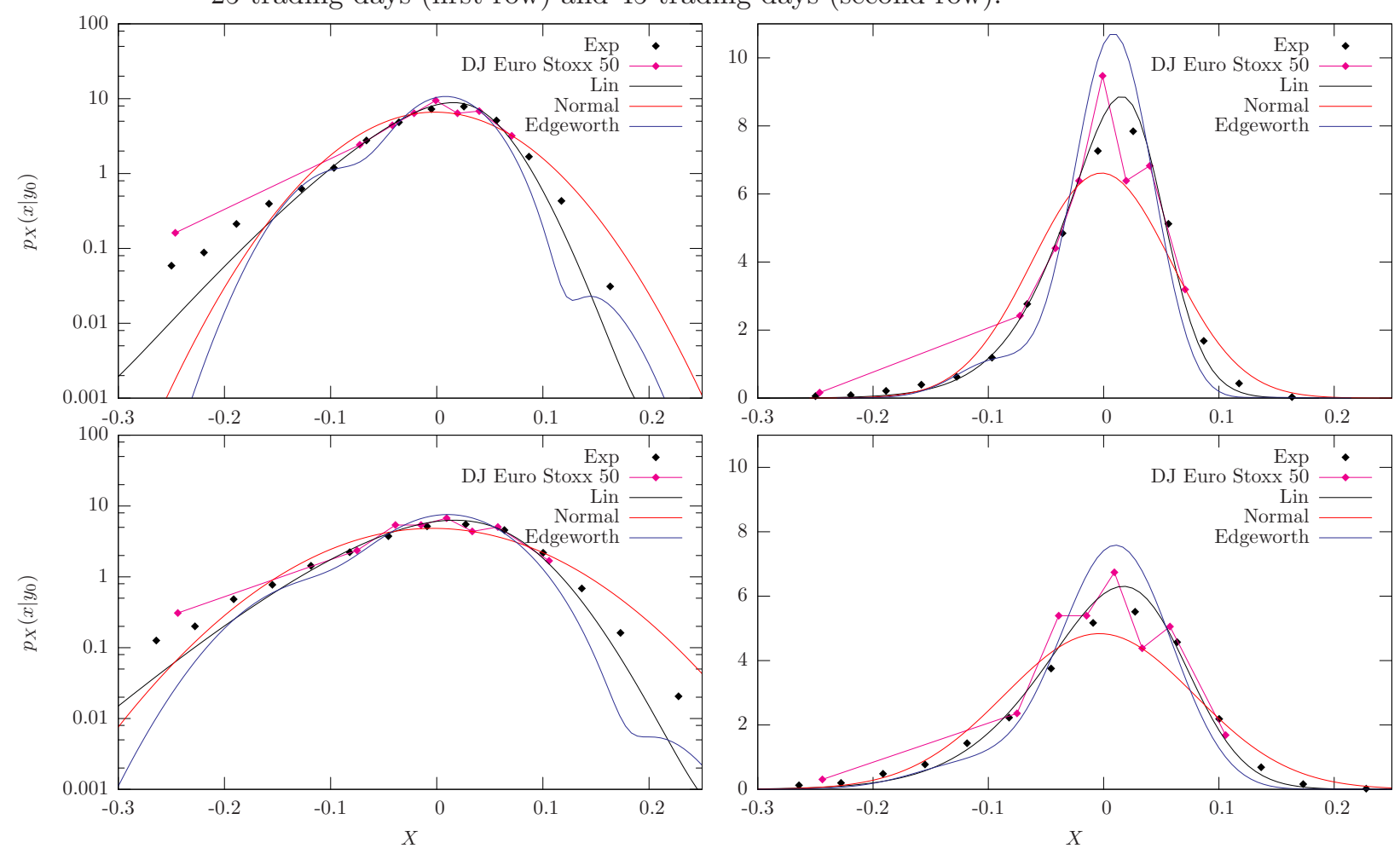

Before concluding, it should be emphasized that further considerations about the capability of the linear model to reproduce known financial evidences could be derived by comparing the theoretical predictions of the model for the fair prices of options with the corresponding market option values. Actually, since our model has been mainly developed having in mind the time evolution of risk neutral distributions, this would allow to derive implied distribution functions over a given time horizon to be compared with the expectations of the model. For example, in [18, 44], it is shown that the implied distribution functions corresponding to options with a time to maturity of one month have an empirical kurtosis value of the order 1, in good agreement with the model predictions for $\beta$ values in the range $5 \% \div 10 \%$. This kind of analysis, which could be performed along the lines of [45], is presently in progress and is left to a future publication. 


\section{Conclusions and perspectives}

In this paper we have dealt with the problem of the analytical characterization of the probability distribution of returns under an exponential Ornstein-Uhlenbeck dynamics for volatility. Such processes have been widely studied, in particular in the econophysics literature, from the point of view of multi-time scale properties, leverage effect and stationary volatility distribution [18, 20, 21, 22, 25, 26]. However, to the best of our knowledge, a systematic approach to the study of returns distribution is missing in literature. Our interest is referred to the possibility of a financial application in the context of option pricing of a closed-form expression, even if approximated, for the probability distribution. The first attempt to obtain such a result can be found in [25] under the hypothesis discussed in Section 2, in particular under the assumption of a stationary regime for the hidden $Y$ process. We have generalized that result to the out-of-stationary regime, with the same constraint of the amplitude of volatility fluctuations, $k$, higher than its normal level, $m(\lambda=k / m \gg 1)$. Indeed, we have provided a closed-form expression in terms of the Edgeworth expansion. This is one of the main results of our work. We have strongly tested our analytical result with MC simulations of the discrete Euler-Maruyama scheme of Eq. (2) and Eq. (5). The goodness of the approximation has been checked with a careful analysis of the discretization step and $\mathrm{MC}$ number of paths for a quite reasonable choice of model parameters. We have found a good agreement for low values of $\beta$, the stationary variance of the log-volatility, while our results worsen when $\beta$ increases even if $\lambda$ increases too. For this reason in Section 4 we have explored the scenario opened by low $\beta$ values which allows us to expand the exponentials involved in the model up to first order in $Y$. Following the technique pioneered by Heston in [13], we have solved exactly the Fokker-Planck backward equation associated with the linear model by means of a suitable trial solution. The full expression is reported in Section 4 and is the main result of this work. Eq. (37)Eq. (39) are quite involved and require some cares, as stressed in the text. Indeed they naturally arise in a complex domain and the presence of multi-valued square root and logarithmic function introduces pain and angers of branching effects. They have been tackled following suggestions coming from the literature related to the Heston model. In particular, we have verified the smoothness of the real and imaginary part of the characteristic function for the parameters we consider. The final task of Fourier anti-transformation has been accomplished by means of FFT techniques. We have numerically tested the effectiveness of the analytical expression and we have found a perfect agreement. Moreover, the agreement between the results of the MC simulation of the discrete scheme of both the complete and linear model make us confident of the numerical convergence of the exponential process for low $\beta$ values. The last section of this paper has been devoted to the comparison of analytical predictions with real market data. The results of our analysis, in particular those for the German DAX30 and the Dow Jones Euro Stoxx 50 indexes, confirm the capability of the linear model to capture the statistical properties of the returns distribution for moderate values of $\beta$ and over 
appropriate time horizons.

As future development we plan to show how the SV model we have studied in this work can emerge in a quite natural way in the context of option pricing and risk management. In particular, we will discuss how to construct a hedged portfolio with associated underlying's dynamics following the exponential Ornstein-Uhlenbeck model. The knowledge of a closed-form expression for the characteristic function allows to implement a Carr - Madan-like approach and to calibrate the Heston-like option price formulae over the implied volatility surfaces. Moreover, we are interested in performing a numerical comparison between option prices obtained through full MC evaluation, our analytical formulae based on a linear model and other closed-form expressions available in literature [29, 46].

\section{Acknowledgments}

We would like to thank D.Delpini and F.Piccinini for valuable discussion and helpful assistance in the later stage of this work. We also acknowledge the anonymous referee for useful comments.

\section{Appendix A. Derivation of $A\left(\omega_{1}, y_{0}, \tau\right), B\left(\omega_{1}, y_{0}, \tau\right)$ and $C\left(\omega_{1}, y_{0}, \tau\right)$}

In order to derive approximate relations for the functions $A, B$ and $C$, we substitute the ansatz (13) in Eq. (12). From relation (14) we argue that relevant information correspond to small $\omega_{2}$ regime and moreover we look for a solution valid in large $\lambda$ regime. Expanding the exponentials up to order $1 / \lambda$ and equating the coefficients of $\omega_{2}$, $\omega_{2}^{2}$ and terms independent of $\omega_{2}$, we find the following first order ODEs

$$
\begin{aligned}
& \dot{A}=-2 \theta\left(\omega_{1}\right) A+\frac{1}{2} \lambda^{2}, \\
& \dot{B}=-\theta\left(\omega_{1}\right) B+\frac{2 i \omega_{1}^{2}}{\lambda} A-\frac{i \alpha \gamma \lambda}{k^{2}}+\lambda \rho \omega_{1}, \\
& \dot{C}=\frac{i \omega_{1}^{2}}{\lambda} B+\frac{1}{2} \omega_{1}^{2}+\frac{i \omega_{1}}{2 \lambda}
\end{aligned}
$$

with

$$
\theta\left(\omega_{1}\right) \doteq \frac{1}{2 \beta}-i \rho \omega_{1}
$$

The dot denotes a derivative w.r.t. $\tau$. The initial condition (11) traduces in

$$
\begin{aligned}
& A\left(\omega_{1}, y_{0}, 0\right)=0, \\
& B\left(\omega_{1}, y_{0}, 0\right)=-i \lambda y_{0}, \\
& C\left(\omega_{1}, y_{0}, 0\right)=0 .
\end{aligned}
$$

Solutions of equations (A.1), (A.2) and (A.3) with boundary conditions (A.5), (A.6) and (A.7) read respectively

$$
A\left(\omega_{1}, y_{0}, \tau\right)=\frac{\lambda^{2}}{4 \theta\left(\omega_{1}\right)}\left[1-\mathrm{e}^{-2 \theta\left(\omega_{1}\right) \tau}\right],
$$




$$
\begin{aligned}
B\left(\omega_{1}, y_{0}, \tau\right)= & \lambda\left[-i y_{0} \mathrm{e}^{-\theta\left(\omega_{1}\right) \tau}+\frac{i \omega_{1}^{2}}{2 \theta^{2}\left(\omega_{1}\right)}\left(1-\mathrm{e}^{-\theta\left(\omega_{1}\right) \tau}\right)^{2}\right. \\
& \left.+\frac{1}{\theta\left(\omega_{1}\right)}\left(\rho \omega_{1}-\frac{i \alpha \gamma}{k^{2}}\right)\left(1-\mathrm{e}^{-\theta\left(\omega_{1}\right) \tau}\right)\right], \\
C\left(\omega_{1}, y_{0}, \tau\right)= & \frac{i \omega_{1}}{2 \lambda} \tau+\frac{\omega_{1}^{2}}{2} \tau \\
& +i \omega_{1}^{2}\left[-i y_{0} \frac{1-\mathrm{e}^{-\theta\left(\omega_{1}\right) \tau}}{\theta\left(\omega_{1}\right)}\right. \\
& +\frac{i \omega_{1}^{2}}{2 \theta^{2}\left(\omega_{1}\right)}\left(\tau-2 \frac{1-\mathrm{e}^{-\theta\left(\omega_{1}\right) \tau}}{\theta\left(\omega_{1}\right)}+\frac{1-\mathrm{e}^{-2 \theta\left(\omega_{1}\right) \tau}}{2 \theta\left(\omega_{1}\right)}\right) \\
& \left.+\frac{1}{\theta\left(\omega_{1}\right)}\left(\rho \omega_{1}-i \frac{\gamma}{2 \beta}\right)\left(\tau-\frac{1-\mathrm{e}^{-\theta\left(\omega_{1}\right) \tau}}{\theta\left(\omega_{1}\right)}\right)\right] .
\end{aligned}
$$

\section{References}

[1] Bachelier 1900 Ann. Sci. École Norm. Sup. 17 21-86

[2] Einstein A 1905 Ann. Phys. 17 549-560

[3] von Smoluchowski M Ann. Phys. 21 756-780

[4] Langevin P 1908 C. R. Acad. Sci. 146 530-533

[5] Uhlenbeck G E and Ornstein L S 1930 Phys. Rev. 36 823-841

[6] Kramers H A 1940 Physica 7 284-304

[7] Risken H The Fokker-Planck equation: Methods of Solution and Applications (Springer)

[8] Brigo D and Mercurio F 2006 Interest Rate Models - Theory and Practice: with Smile, Inflation and Credit (Berlin Heidelberg New York: Springer)

[9] Black F and Scholes M 1973 J. Polit. Economy 72 637-659

[10] Scott L 1987 J. Finan. Quant. Anal. 419-438

[11] Hull J and White A 1987 J. Finance 42 281-300

[12] Stein E and Stein J 1991 Rev. Finan. Stud. 4 727-752

[13] Heston S L 1993 Rev. Finan. Stud. 6 327-343

[14] Ball C and Roma A 1994 J. Finan. Quant. Anal. 29 589-607

[15] Fouque J P, Papanicolaou G and Sircar K R 2000 Derivatives in Financial Markets with Stochastic Volatility (Cambridge University Press)

[16] Carr P and Madan D 1999 J. Comput. Finance 2 61-73

[17] Lewis A L 2001 A simple option formula for general jump-diffusion and other exponential Lévy processes (Available at SSRN: http://ssrn.com/abstract=282110)

[18] Bouchaud J P and Potters M 2000 Theory of Financial Risk and Derivative Pricing: from Statistical Physics to Risk Management (Cambridge University Press)

[19] Bacry E, Delour J and Muzy J F 2001 Phys. Rev. E 64026103

[20] Dragŭlescu A A and Yakovenko V M 2002 Quant. Finance 2 443-453

[21] Masoliver J and Perelló J 2002 Int. J. Theoretical Appl. Finance 5 541-562

[22] Masoliver J, Perelló J and Anento N 2004 Physica A 344 134-137

[23] Borland L, Bouchaud J P, Muzy J F and Zumbach G 2005 Wilmott

[24] Borland L and Bouchaud J P 2005 On a multi-timescale statistical feedback model for volatility fluctuations Preprint physics soc-ph/0507073

[25] Masoliver J and Perelló J 2006 Quant. Finance 6 423-433

[26] Cisana E, Fermi L, Montagna G and Nicrosini O 2007 A comparative study of stochastic volatility models Preprint physics.soc-ph/0709.0810

[27] Bonanno G, Valenti D and Spagnolo B 2007 Phys. Rev. E 75 016106-016108 
[28] Valenti D, Spagnolo B and Bonanno G 2007 Physica A 382 311-320

[29] Perelló J, Sircar R and Masoliver J 2008 Option pricing under stochastic volatility: the exponential Ornstein-Uhlenbeck model Preprint physics.soc-ph/0804.2589

[30] Fouque J P, Papanicolaou G and Sircar K R 2000 Int. J. Theoretical Appl. Finance 3 101-142

[31] Bouchaud J P, Matacz A and Potters M 2001 Phys. Rev. Lett. 87228701

[32] Masoliver J, Perelló J and Bouchaud J P 2004 Appl. Math. Finance 11 27-50

[33] Bormetti G and Cazzola V In preparation

[34] Gardiner C W 1983 Handbook of Stochastic Methods (Berlin and New York: Springer-Verlag)

[35] Cramér H 1951 Mathematical Methods of Statistics (Princeton: Princeton University Press)

[36] Jondeau E, Poon S H and Rockinger M 2007 Financial Modeling Under Non-Gaussian Distributions (Springer)

[37] Glasserman P 2003 Monte Carlo Methods in Financial Engineering (Springer)

[38] Adams J, Brom T and Layton J 2007 Microwulf: Breaking the 100 USD/GFLOP Barrier Available at: http://www.clustermonkey.net

[39] Albrecher H, Mayer P, Schoutens W and Tistaert J 2007 Wilmott 83-92

[40] Kahl C and Jäckel P 2005 Wilmott 94-103

[41] Lord R and Kahl C 2006 Why the Rotation Count Algorithm Works (Tinbergen Institute Discussion Paper)

[42] Pasquini M and Serva M 2000 Eur. Phys. J. B 16 195-201

[43] Mantegna R and Stanley H E 2000 An Introduction to Econophysics: Correlations and Complexity in Finance (Cambridge University Press)

[44] Potters M, Cont R and Bouchaud J 1998 Europhys. Lett. 41 239-244

[45] Duffie D, Pan J and Singleton K 2000 Econometrica 68 1343-1376

[46] Filho G B and Rosenfeld R 2004 Physica A 344 484-490 